\title{
On arc-polarized structures in the solar wind
}

\author{
B. U. Ö. Sonnerup ${ }^{1}$, S. E. Haaland ${ }^{2,3}$, and G. Paschmann ${ }^{4}$ \\ ${ }^{1}$ Thayer School of Engineering, Dartmouth College, Hanover, New Hampshire, USA \\ ${ }^{2}$ Max Planck Institut für Sonnensystemforschung, Katlenburg-Lindau, Germany \\ ${ }^{3}$ Department of Physics and Technology, University of Bergen, Bergen, Norway \\ ${ }^{4}$ Max Planck Institut für extraterrestrische Physik, Garching, Germany
}

Received: 23 March 2010 - Accepted: 17 May 2010 - Published: 8 June 2010

\begin{abstract}
A theoretical model is proposed to account for some of the behavior of arc-polarized magnetic structures seen in the solar wind. To this end, an exact analytical solution is developed that describes infinite plane wave trains of arbitrary amplitude in a plasma governed by ideal Hall MHD. The main focus is on intermediate-mode wave trains, which display double-branched magnetic hodogram signatures similar to those seen in the solar wind. The theoretically derived hodograms have field rotation in the ion-polarized sense at a slightly depressed field magnitude on one branch and an electron-polarized rotation at a slightly enhanced field magnitude on the other branch. The two branches are joined at the two "turning points", at which the normal flow is exactly Alfvénic. The behavior is accounted for in terms of the opposite dispersive properties of ion and electron whistlers. The hodograms derived from the theory are shown to compare favorably with those of one event, observed by the Cluster spacecraft near the ecliptic plane, and one event at high heliographic latitude observed by the Ulysses spacecraft. However, these two observed structures comprise only a single full wave period, approximately from one turning point to the other and then back again. The theory can be used to predict propagation direction (away from, or towards, the sun) from magnetic data alone, provided the sign of the magnetic field component along the wave normal can be reliably determined. Under the same condition, it also predicts whether the ion-polarized branch should precede or follow the electronpolarized branch. Both behaviors are seen in the solar wind. The major shortcoming of the theory is that it fails to reproduce the observed saw-tooth like time series for the magnetic field, in which the field rotation is rapid in the ion sense and slow in the electron sense. Instead, the theory gives about the same rotation rates. Possible explanations for this dis-
\end{abstract}

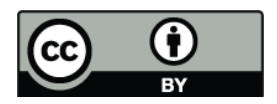

Correspondence to: B. U. Ö. Sonnerup (sonnerup@dartmouth.edu) crepancy are discussed. Also discussed is the fact that the magnetic field measurements by Cluster, while giving high quality determinations of normal direction and normal field component for each of the four spacecraft, indicate a reversal of the normal field component and the predicted propagation sense during the event, as well as a wide spread in the four normal vector orientations.

Keywords. Interplanetary physics (Solar wind plasma) Space plasma physics (Kinetic and MHD theory)

\section{Introduction}

The purpose of this paper is to propose a theoretical description of what we will call "double-arc polarized" magnetic structures observed in the solar wind (Lichtenstein and Sonett, 1980; Tsurutani et al., 1994, 1996, 1997; Riley et al., 1996; Tsurutani and Ho, 1999; Horbury and Tsurutani, 2001, and references therein). In these structures, the magnetic field appears to be tipping back and forth, roughly speaking in a plane and with approximately constant field magnitude, with a small field component normal to that plane, i.e., along the propagation direction, being present as well. The formation of such structures, starting from linearly polarized Alfvén waves near the sun and evolving as they are carried outward by the solar wind, has been extensively studied, both analytically and by use of numerical simulations. Here we attempt to find steady state, one-dimensional (1D: $\partial / \partial y=\partial / \partial z=0)$, Hall-MHD solutions for large amplitude wave trains of this type. The attempt is partially successful in the sense that solutions are obtained in which the tangential magnetic field tips back and forth with nearly, but not precisely, constant magnitude, as observed. However, a second important aspect of the observations is not contained in our simple 1-D model, namely rapid rotation of the field in one sense, followed by, or preceded by, slow rotation in the

Published by Copernicus Publications on behalf of the European Geosciences Union. 


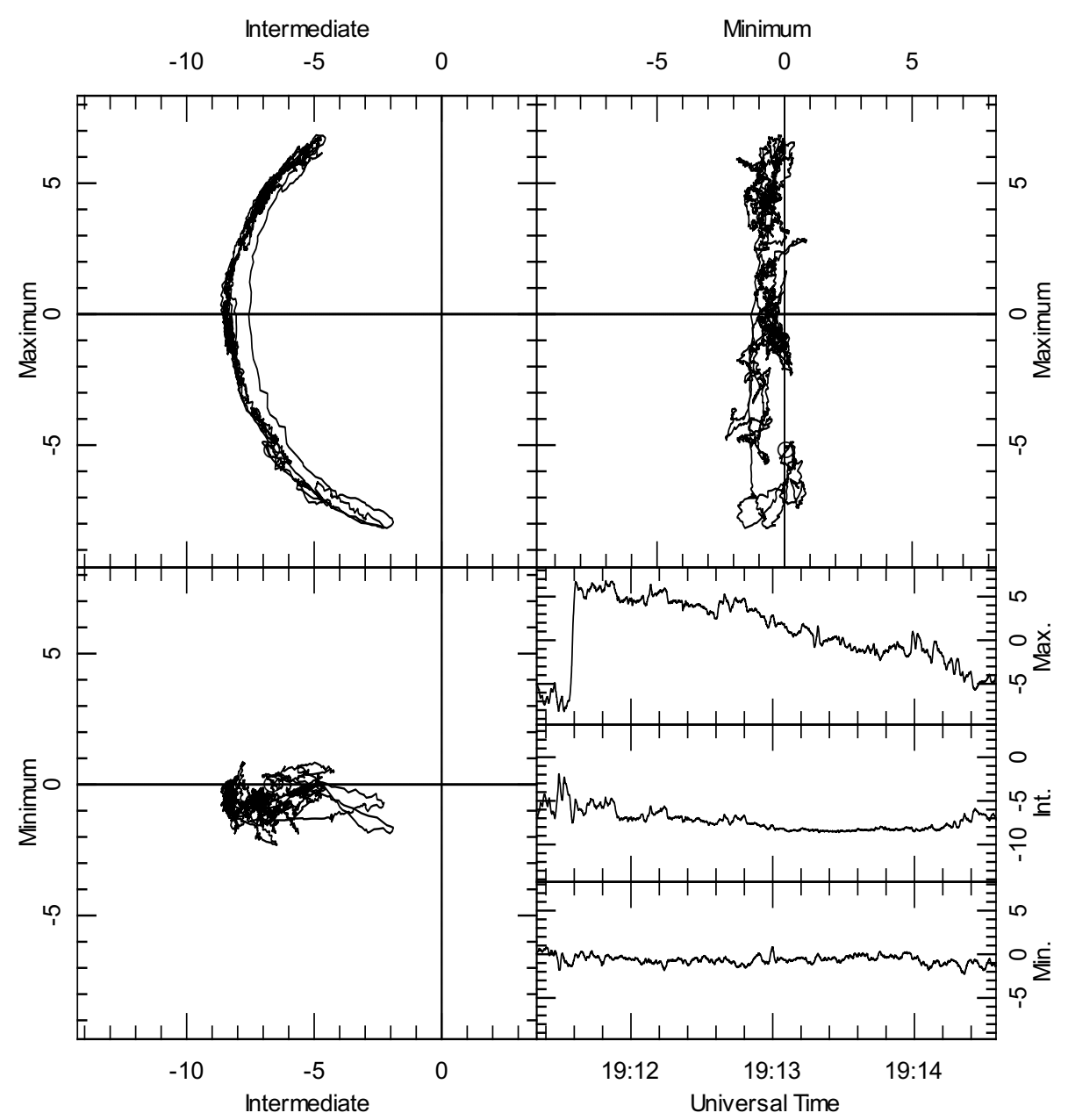

Time Interval (UT): 2003-02-03 19:11:20.092 - 19:14:34.503

Fig. 1. Magnetic hodogram for Cluster 1 double-arc polarized event, seen in the solar wind on 3 February 2003 , at $(16.85 ; 9.15 ;-1.77) R_{\mathrm{E}}$ (GSE). Hodogram curve starts at small circle. It is shown in three projections, using as axes the right-handed orthonormal eigenvector triad $\left(\hat{\boldsymbol{x}}_{1} ; \hat{\boldsymbol{x}}_{2} ; \hat{\boldsymbol{x}}_{3}\right)$ (the principal axes) from variance analysis of the magnetic field (MVAB). Here $\hat{\boldsymbol{x}}_{1}=(0.9336 ; 0.2936 ; 0.2057)(\mathrm{GSE})$ is the minimum variance direction, with variance $\lambda_{1}=0.26 \mathrm{nT}^{2} ; \hat{\boldsymbol{x}}_{2}=(0.0599 ; 0.4380 ;-0.8970)$ is the intermediate variance direction, with $\lambda_{2}=1.41 \mathrm{nT}^{2}$; and $\hat{\boldsymbol{x}}_{3}=(-0.3534 ; 0.8497 ; 0.3914)$ is the maximum variance direction, with $\lambda_{3}=14.50 \mathrm{nT}^{2}$. Note that our ordering of the eigenvectors differs from that used by Sonnerup and Scheible (1998). Time series of field components along the principal axes are shown in the bottom right panel. Note the saw-tooth like behavior of the curve for the maximum-variance component. Time resolution is 22.4 samples/s.

opposite sense, the latter with superimposed Alfvénic fluctuations. In our model, the rotation rates for the two senses instead turn out to be approximately the same. Possible reasons for this defect will be discussed. By examination of two particularly well-organized events, one seen by the four Cluster spacecraft and one by the Ulysses spacecraft, we will show that other aspects of our model are capable of accounting for their observationally obtained counterparts.

The magnetic field behavior in a double-arc polarized structure, seen by Cluster 1 in the near-earth solar wind but outside the region influenced by the bow shock, is shown in Fig. 1. In this figure, the magnetic field is presented as three magnetic hodogram projections and also, to the lower right in the figure, as time series of the field components in the maximum, intermediate, and minimum variance directions, obtained from standard minimum variance analysis of the field (MVAB; see the review by Sonnerup and Scheible, 1998). The rapid reversal of the maximum-variance field component, followed by a much slower return to more or less the original direction, is seen in the uppermost time series. The rotation of the field in the tangent plane of the structure is shown in the top left hodogram projection. 
a)

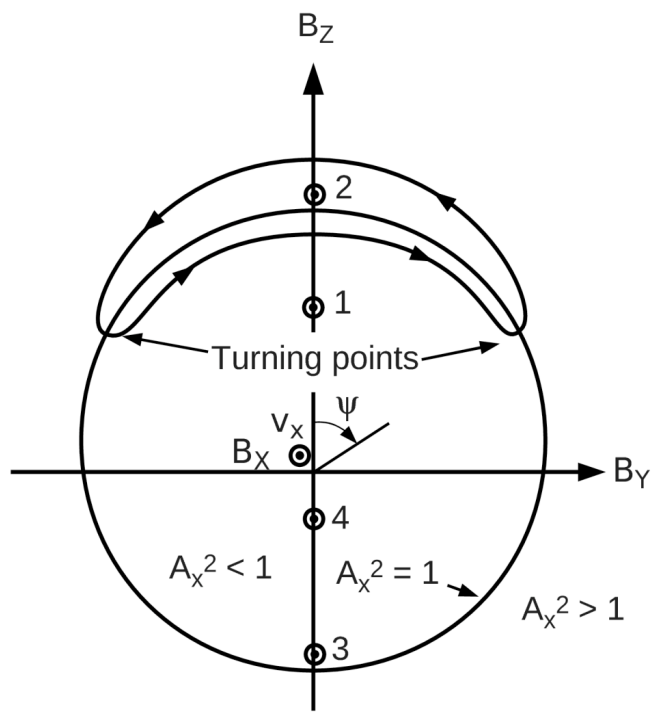

b)

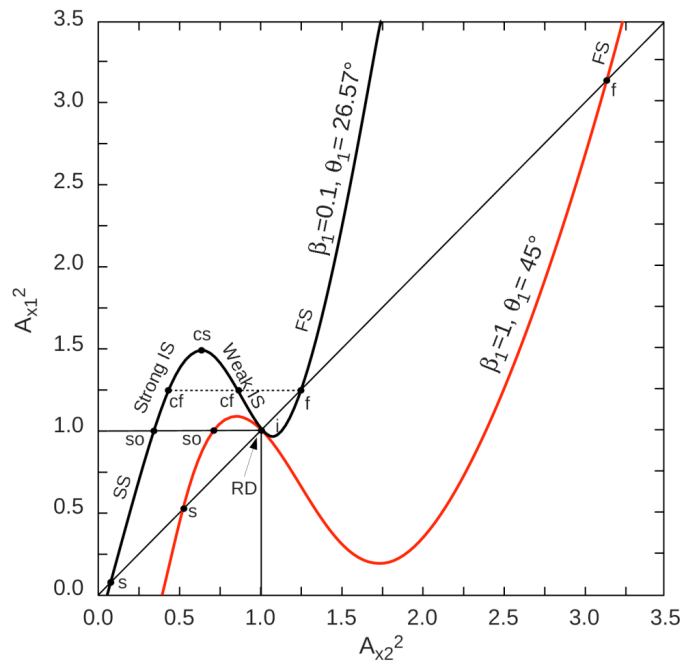

Fig. 2. MHD shock properties. (a) Schematic drawing, showing the location of fixed points in the tangential hodogram plane. These four points represent the possible upstream and downstream states of fast shocks $(1 \rightarrow 2)$, weak and strong subfast intermediate shocks $(2 \rightarrow 3$, or $2 \rightarrow 4)$, and slow shocks $(3 \rightarrow 4)$. Superfast intermediate shocks $(1 \rightarrow 3$ and $1 \rightarrow 4)$ can also occur. Also shown is a banana-shaped hodogram trace, around the fixed point 2, for a non-dissipative wave train. This trace marks the successive locations, as the normal coordinate $x$ increases, of the head of an arrow from the origin, representing the tangential magnetic field vector. The indicated sense of motion around the banana is for the case where $\left(v_{\mathrm{X}} / B_{\mathrm{X}}\right)$ is positive. (b) Representation of shock jump conditions in terms of upstream $\left(A_{\mathrm{X} 1}\right)$ and downstream $\left(A_{\mathrm{x} 2}\right)$ Alfvén numbers for two different sets of values of upstream plasma beta, $\beta_{1}$, and angle, $\theta_{1}$, between shock normal and upstream field. Acronyms FS, IS, and SS represent fast, intermediate, and slow shocks. The symbols $s, i$, and $f$, denote small-amplitude slow, intermediate, and fast modes; the switch-off shock is denoted by so. Superfast intermediate shocks occur above the line $c f$-cf-f (after Hau and Sonnerup, 1989).

The rapid initial rotation is seen to occur at a slightly depressed field magnitude, while the slow return rotation has a slightly enhanced field magnitude. The slow branch of the hodogram also shows more rapid, but smaller amplitude, field rotations back and forth along the hodogram path, associated with substantial fluctuations of the field component in the normal (minimum-variance) direction, as seen in the upper right hodogram projection. A main hypothesis underlying our model is that the two branches of the tangential hodogram should be considered as part of one and the same structure and not as two unrelated field rotations. We call the events "double-arc" polarized because the simpler term "arcpolarized" is ambiguous: it can also refer to observed field rotations of only one sense at nearly constant field magnitude (so-called rotational discontinuities, or RDs for short). We will refer to such events as being "single-arc" polarized. The double-arc structures will be described as one full period of a highly non-linear wave train. The single-arc polarized structures correspond to field rotation, either along the inner or the outer branch. Multi-arc polarized structures can in principle also occur, although observations of such structures seldom show sufficiently well organized behavior to argue that they represent two or more periods of one and the same periodic wave train.
The main objective of our study is to develop a simple, Hall-MHD based, model of wave trains that accounts for the double-branched nature of the tangential hodogram and for the fact that the slow rotation sometimes follows the rapid rotation, as in Fig. 1, while in other events it may precede the fast rotation. The slow-fast order was seen in a Ulysses event frequently discussed in the literature (see, e.g., Fig. 10 in Tsurutani and Ho, 1999, and also Fig. 8 of the present paper). Tsurutani and Ho have argued that the fast rotation is the result of phase steepening within this part of the wave, caused by dispersive effects. This is one of several reasons to include Hall physics in our model.

Our presentation is organized as follows: in Sect. 2, we develop the theory and provide numerical examples; in Sects. 3 and 4 , we compare the theoretical predictions with our two observed events. The main results and conclusions are summarized and discussed in Sect. 5. 


\section{Theory}

\subsection{Background}

To set the stage for our analysis, we begin by briefly discussing the magnetic structure of time-independent, dispersive magneto-hydrodynamic shocks. They can be described in terms of the behavior of the two field components, $B_{\mathrm{y}}$ and $B_{\mathrm{Z}}$, tangential to the shock surface (a normal component, $B_{\mathrm{X}}$, is also present and remains constant throughout the structure). In a plot of $B_{\mathrm{z}}$ versus $B_{\mathrm{y}}$ (a tangential magnetic hodogram), shock structures are described as, sometimes complicated, transitions (paths) between pairs of "fixed points", also called "stationary points", that represent their upstream and downstream states. It follows from the coplanarity condition, valid for shocks, that these points can all be placed on, for example, the $B_{\mathrm{z}}$-axis of the hodogram plane. As illustrated in Fig. 2a, there are four fixed points; two on the positive and two on the negative $B_{\mathrm{Z}}$-axis. Points 1 and 2 , both located on the positive $B_{\mathrm{Z}}$-axis by choice, are the upstream and downstream states of fast shocks. Point 2 is also the upstream state of weak and strong but subfast intermediate shocks (the superfast version has point 1 as its upstream state), for which the downstream states are points 3 and 4 , respectively, both located on the negative $B_{\mathrm{Z}}$-axis, indicating that the tangential field reverses sign in intermediate shocks. Points 3 and 4, respectively, are also the upstream and downstream states of a slow shock. A graphical representation of the relationship between upstream and downstream Alfvén-Mach numbers for these various shocks was developed by Hau and Sonnerup (1989) and is, for convenience, reproduced in Fig. 2b. In this plot, we note that the strongest slow shock, called the switch-off shock because it brings the tangential field to zero $\left(B_{\mathrm{y}}=B_{\mathrm{z}}=0\right)$, marks the change from a slow shock to a strong intermediate shock and that the infinitely weak intermediate shock becomes a rotational discontinuity, across which the plasma state and field magnitude remain unchanged; in that limit, co-planarity is no longer required. The fixed points themselves can be spiral points, nodes, or saddle points. Furthermore, the hodogram plane can be thought of as having two layers: it has a supersonic and a subsonic "Riemann sheet" on which the plasma flow component in the x-direction, relative to the structure, is supersonic or subsonic, respectively. We emphasize that it is only the flow component along the normal (x-) direction that matters; the total flow may well be supersonic. The fixed points do not all lie on the same Riemann sheet. Point 1 is always on the supersonic sheet and point 4 is always on the subsonic sheet but, depending on parameter values, points 2 and 3 may be on either sheet. The two sheets come together along a closed curve in the hodogram plane. On this curve, the flow component along the shock normal is sonic. If the shock dissipation is described as purely resistive, i.e., if viscosity and heat conduction are neglected, transitions from supersonic to subsonic flow along the $\mathrm{x}$-direction occur as dis- continuous jumps from the supersonic to the subsonic Riemann sheet, across which the jump conditions are those of an ordinary gas-dynamic shock. These various shock structures were described by the resistive models, analyzed by Hau and Sonnerup $(1989,1990)$, for ordinary MHD as well as for Hall MHD.

In the work to be presented here, we will examine structures governed by Hall MHD (electron inertia is not included and, we believe, not needed) in the limit where the resistivity and all other dissipative coefficients have been set to zero. In that case, fixed points of the spiral variety become converted to "centers" around which the hodogram curves form a set of nested closed loops. These loops describe wave trains of various angular amplitudes. With our application to doublearc polarized structures in the solar wind in mind, we will focus attention on orbits around the fixed point 2 , and assume it to be located on the subsonic Riemann sheet, as is indeed the case in our applications. For an example, see the schematic plot in Fig. 2a. These wave trains should be identified as being of the intermediate mode. However, the general solution we will present applies to fast-mode and slow-mode finite amplitude wave trains as well. Solitary waves, in which the tangential field rotates by exactly $360^{\circ}$, are described by curves (separatrices) in the hodogram plane that mark the boundary between different classes of solutions (see Fig. 3a). Finally, note that in order to compare the observed tangential hodogram in Fig. 1 with the theoretically derived versions in Fig. 3, the latter will need to be rotated counter-clockwise by $90^{\circ}$.

\subsection{Basic equations}

The development is based on ideal Hall MHD. The ion and electron pressures are assumed isotropic and the flow is compressible but isentropic (or polytropic). The analysis is performed in a frame of reference traveling with the wave train in the negative $\mathrm{x}$-direction and having vanishing electric field components in the tangent $(\mathrm{y}-\mathrm{z})$ plane. In this frame of reference, an intrinsic electric field component in the $\mathrm{x}$-direction remains as part of the wave structure but does not enter into the main description. The governing equations can be obtained from the three principal equations, numbered (6), (7), and (8), in the work by Hau and Sonnerup (1990) on the structure of resistive-dispersive shocks, by simply letting the resistivity $\eta$ approach zero. The procedure is to let their parameter $h \equiv B_{\mathrm{x}} / n e \eta$ approach infinity. After simple algebraic rearrangements, the principal equations for the tangential field, expressed in the coordinate system we described in the previous section, then become

$$
\begin{aligned}
& \lambda_{i 2} \frac{A_{\mathrm{x}}^{2}}{A_{\mathrm{x} 2}} \frac{d B_{\mathrm{y}}}{d x}=-\left(A_{\mathrm{x}}^{2}-1\right) B_{\mathrm{z}}+\left(A_{\mathrm{x} 2}^{2}-1\right) B_{\mathrm{z} 2} \\
& \lambda_{i 2} \frac{A_{\mathrm{x}}^{2}}{A_{\mathrm{x} 2}} \frac{d B_{\mathrm{z}}}{d x}=\left(A_{\mathrm{x}}^{2}-1\right) B_{\mathrm{y}}
\end{aligned}
$$


a) Theory: Numerical example

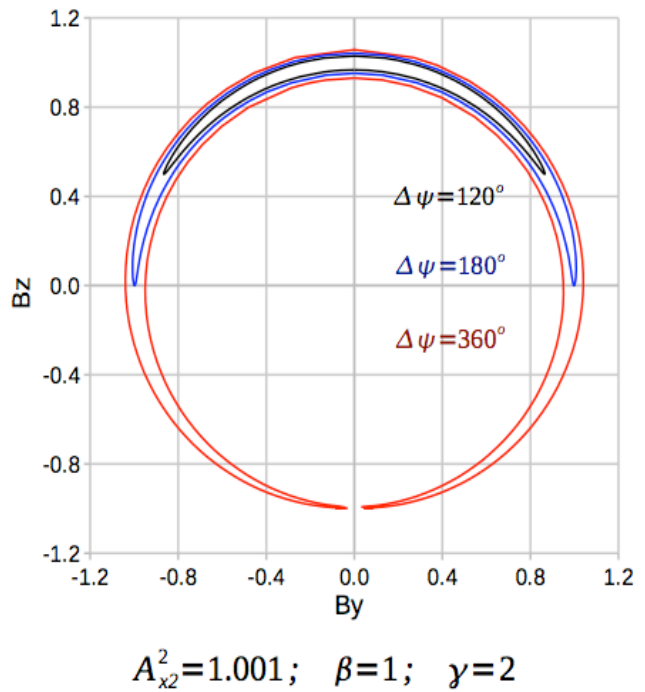

b) Theory: Cluster 1 event

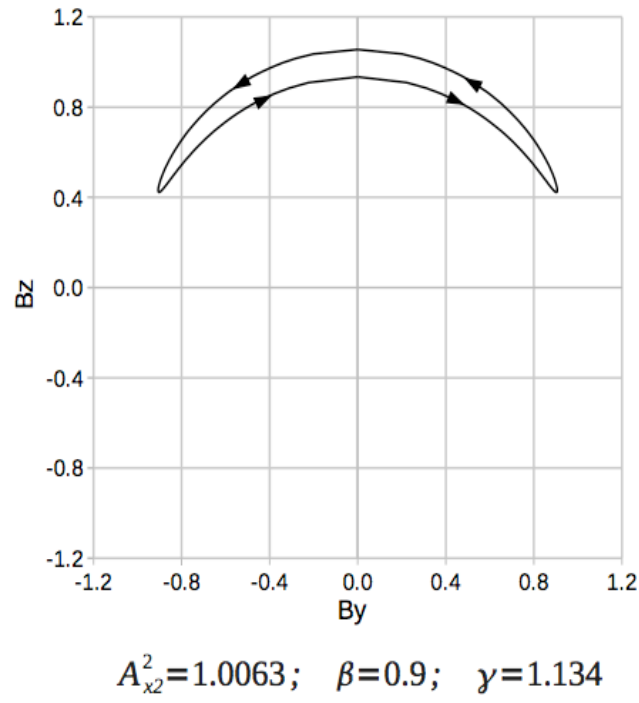

Fig. 3. Examples of tangential hodograms from theory. (a) Hodogram for parameter values at fixed point 2 given by $\beta_{2}=1, A_{\mathrm{x} 2}^{2}=1.001$, and with ratio of specific heats $\gamma=2$. On the blue curve, the field rotates by $\Delta \psi=180^{\circ}$ from the left to the right turning point, at both of which $B_{\mathrm{Z}}^{*}=0$, and then by $\Delta \psi=-180^{\circ}$ back again. For the black curve, $\Delta \psi=120^{\circ}$ instead, and for the red curve, $\Delta \psi=360^{\circ}$. The red curves are separatrices in the hodogram; they intersect at the fixed point 3 (a saddle point) and represent left and right polarized solitary waves. (b) Hodogram for parameter values representative of the Cluster 1 event $\left(\beta_{2}=0.9 ; \theta_{2}=86.2^{\circ} ; B_{\mathrm{z}}^{*} / B_{\mathrm{z} 2}=\sin 25^{\circ}\right)$, with the values $A_{\mathrm{x} 2}^{2}=1.0063$ and $\gamma=1.134$ chosen to reproduce the observed separation (Fig. 1) of the inner and outer branch of the hodogram at the symmetry point $\left(B_{\mathrm{y}}=0\right)$ as well as the observed spatial extent of the inner hodogram branch. Arrowheads indicate the sense of field rotation that corresponds to $v_{\mathrm{X}} / B_{\mathrm{X}}>0$.

These two equations are straightforward to derive. They express the tangential components of the generalized Ohm's law, combined with the integrated tangential momentum equations to eliminate the tangential velocity components, and mass conservation; the quantity $\lambda_{i 2}=\left(m_{i} / \mu_{0} n_{2} e^{2}\right)^{1 / 2}$ is the ion inertial length, evaluated at the fixed point 2 , which is a 'center' located at $\left(0, B_{\mathrm{z} 2}\right)$ in the hodogram plane, around which our hodogram trajectories will appear as a nested set; the local Alfvén-Mach number for the axial flow is $A_{\mathrm{X}}=\left(v_{\mathrm{x}} / B_{\mathrm{X}}\right)\left(\mu_{0} n m_{i}\right)^{1 / 2}$, which has the value $A_{\mathrm{x} 2}$ at the fixed point. We have assumed $B_{\mathrm{z} 2}$ to be a positive quantity but emphasize that $A_{\mathrm{x}}$ will be positive or negative depending on whether $v_{\mathrm{x}}$ and $B_{\mathrm{x}}$ have equal or opposite signs. Equation (8) in the work by Hau and Sonnerup (1990) describes the behavior of $A_{\mathrm{x}}^{2}$. After lengthy algebra, it is obtained from the energy equation, combined with the three momentum equations to eliminate pressure and tangential velocity components, and mass conservation. In the Hau-Sonnerup version of the equation, we will, for later convenience, use $B^{2}=B_{\mathrm{t}}^{2}+B_{\mathrm{x}}^{2}$ and $B_{\mathrm{z} 2} / B_{\mathrm{x}}=\tan \theta_{2}$ to eliminate $B^{2}$ and $B_{\mathrm{x}}$ in favor of the tangential field $B_{\mathrm{t}}=\left(B_{\mathrm{y}}^{2}+B_{\mathrm{z}}^{2}\right)^{1 / 2}$ and the field angle $\theta_{2}$ at the fixed point. The result is

$A_{\mathrm{x}}^{2}=\frac{\gamma / 2}{\gamma+1}\left[2 A_{\mathrm{x} 2}^{2}+\frac{\beta_{2}}{\cos ^{2} \theta_{2}}+\left(1-\frac{B_{\mathrm{t}}^{2}}{B_{\mathrm{z} 2}^{2}}\right) \tan ^{2} \theta_{2}\right]$

$$
\begin{aligned}
& \pm\left\{\left(\frac{\gamma / 2}{\gamma+1}\right)^{2}\left[2 A_{\mathrm{x} 2}^{2}+\frac{\beta_{2}}{\cos ^{2} \theta_{2}}+\left(1-\frac{B_{\mathrm{t}}^{2}}{B_{\mathrm{z} 2}^{2}}\right) \tan ^{2} \theta_{2}\right]^{2}\right. \\
& +\frac{\gamma-1}{\gamma+1}\left(\tan ^{2} \theta_{2}\right)\left[\frac{B_{\mathrm{t}}^{2}}{B_{\mathrm{z} 2}^{2}}+2\left(A_{\mathrm{x} 2}^{2}-1\right) \frac{B_{\mathrm{z}}}{B_{\mathrm{z} 2}}+\left(A_{\mathrm{x} 2}^{2}-1\right)^{2}\right. \\
& \left.\left.-\frac{A_{\mathrm{x} 2}^{2}}{\sin ^{2} \theta_{2}}\left(A_{\mathrm{x} 2}^{2}+\frac{\gamma \beta_{2}}{\gamma-1}\right)\right]\right\}^{1 / 2}
\end{aligned}
$$

Here the quantity $\gamma=c_{p} / c_{v}$ is the ratio of specific heats at constant pressure and constant volume. Also, $\beta_{2}=$ $p_{2} 2 \mu_{0} / B_{2}^{2}$ is the plasma beta value and $\theta_{2}=\tan ^{-1}\left(B_{\mathrm{z} 2} / B_{\mathrm{x}}\right)$ is the field angle, both evaluated at the fixed point. The normal field component $B_{\mathrm{x}}$ is constant and mass conservation requires $n v_{\mathrm{x}}=$ const., where $n$ is the number density. The \pm sign in front of the square root in Eq. (3) specifies the supersonic (+) and the subsonic (-) Riemann sheets. Sonic conditions occur where the square root vanishes. For our application, the negative sign should be used because we will have $v_{\mathrm{x}}^{2}<\gamma \mathrm{p} / n m_{i}$ in our structures. Also, since point 2 represents the upstream state of an intermediate shock, we must have $A_{\mathrm{x} 2}^{2}>1$, as can be seen in Fig. 2b. If the field magnitude is to remain nearly constant in the wave train, as it is observed to be, then it must belong to the weak intermediatewave family, with $A_{\mathrm{x} 2}^{2}$ only slightly larger than unity. 
Two general comments should be made about Eqs. (1) and (2). First, the space derivatives reverse sign if $A_{\mathrm{x}}$ reverses sign, i.e., if the ratio $v_{\mathrm{X}} / B_{\mathrm{X}}$ reverses sign. Second, the scale length along the $\mathrm{x}$-axis of the wave is of the order of the ion inertial length, unless $A_{\mathrm{x}}^{2}$ is very near unity. This latter situation is assumed to occur in our applications.

\subsection{Turning points}

We note from Eq. (2) that $d B_{\mathrm{z}} / d x=0$ when $A_{\mathrm{x}}^{2}=1$. As an observer moves through the structure toward more positive $\mathrm{x}$-values, the tip of the tangential field vector will be seen to move along the hodogram curve, as illustrated in Fig. 2a. The two locations where $B_{\mathrm{Z}}$ reaches its minimum value, i.e., where $d B_{Z} / d x=0$ and $d^{2} B_{Z} / d x^{2}>0$, are called the turning points. Note that it is the field component $B_{\mathrm{Z}}$ that turns around. The two points on top of the hodogram, where, $B_{\mathrm{y}}=$ 0 , are additional turning points for $B_{\mathrm{Z}}$, but will be referred to as symmetry points instead. There are also turning points for $B_{\mathrm{y}}$, which are located near those for $B_{\mathrm{z}}$. At these latter points, Eq. (1) shows that $\left.B_{\mathrm{z}} / B_{\mathrm{z} 2}=\left(A_{\mathrm{x} 2}^{2}-1\right) /\left(A_{\mathrm{x}}^{2}-1\right)\right)$.

By putting $A_{\mathrm{x}}^{2}=1$ in Eq. (3), the following turning point condition for $B_{\mathrm{Z}}$, denoted by an asterisk, can be obtained after straightforward but lengthy algebra:

$$
\begin{aligned}
\frac{B_{\mathrm{z}}^{*}}{B_{\mathrm{z} 2}}= & \frac{B_{\mathrm{t}}^{* 2} / B_{\mathrm{z} 2}^{2}-1}{2(\gamma-1)\left(A_{\mathrm{x} 2}^{2}-1\right)}+\frac{\gamma}{\gamma-1}+\frac{\gamma \beta_{1} / 2-1}{(\gamma-1) \sin ^{2} \theta_{2}} \\
& +\frac{1}{2}\left(A_{\mathrm{x} 2}^{2}-1\right) \cot ^{2} \theta_{2}
\end{aligned}
$$

As before, we have $B_{\mathrm{t}}^{2} \equiv B_{\mathrm{y}}^{2}+B_{\mathrm{z}}^{2}$. Equation (4) gives the location of the turning points for $B_{\mathrm{Z}}$ in the hodogram plane as a relationship between the non-dimensional "z-coordinate", $B_{\mathrm{Z}} / B_{\mathrm{z} 2}$, in terms of the non-dimensional polar "radius", $B_{\mathrm{t}} / B_{\mathrm{z} 2}$. We see from Eq. (4) that the locus of all points having $A_{\mathrm{x}}^{2}=1$ is a circle with its center on the "z-axis" and slightly displaced above the origin of the hodogram (see Fig. 2a).

As an example, consider the case where $B_{\mathrm{z}}^{*} / B_{\mathrm{z} 2}=0$ so that the two turning points for $B_{\mathrm{Z}}$ are on the $B_{\mathrm{y}}$-axis. This means that the tangential field rotates back and forth by $180^{\circ}$. At the turning points we then have $B_{\mathrm{t}}^{* 2}=B_{\mathrm{y}}^{* 2}$ so that, from Eq. (4), we find them to be at

$$
\begin{aligned}
B_{\mathrm{y}}^{*} / B_{\mathrm{z} 2}= & \pm\left\{1-\left(A_{\mathrm{x} 2}^{2}-1\right)\left[2 \gamma+\left(\gamma \beta_{2}-2\right) / \sin ^{2} \theta_{2}\right.\right. \\
& \left.\left.+(\gamma-1)\left(A_{\mathrm{x} 2}^{2}-1\right) \cot ^{2} \theta_{2}\right]\right\}^{1 / 2}
\end{aligned}
$$

Because $\left(A_{\mathrm{x} 2}^{2}-1\right)$ is small but positive, Eq. (5) shows that, as long as the square bracket remains positive, the turning points will be located slightly inside the circle, given by $B_{\mathrm{t}} / B_{\mathrm{z} 2}=1$. This latter circle is centered at the origin and passes through the fixed point 2. In other words, the hodogram must have nearly semi-circular shape in this example. For other locations of the turning points, Eq. (4) indicates that the hodogram should still look approximately as a circular arc. But, as mentioned already, the hodogram will in reality have two branches that do not precisely overlap but that join at the two turning points. As indicated by Eqs. (1) and (2), and as illustrated in Fig. 2a, there will be an outer branch on which $\left(A_{\mathrm{x}}^{2}-1\right)$ is slightly positive with rotation of the field vector in the hodogram plane of one sense, and an inner branch where $\left(A_{\mathrm{x}}^{2}-1\right)$ is slightly negative with rotation in the opposite sense. At the top of the outer branch (the symmetry point), the two terms on the right-hand side of Eq. (1) have opposite signs, suggesting the possibility of slower rotation along this branch (assuming $A_{\mathrm{x}}>0$, we have $d B_{\mathrm{y}} / d x \leq 0$, because $A_{\mathrm{x}}^{2} \geq A_{\mathrm{x} 2}^{2}$ on this branch). On the inner branch, the two terms have the same sign, both positive, suggesting more rapid rotation in the opposite direction $\left(d B_{y} / d x \geq 0\right)$. However, the difference between the two field rotation rates turns out to be much too small to account for the observed saw-tooth like time series. Except for this fact, the qualitative discussion given above illustrates that our basic equations are capable of producing at least some of the behavior of observed double-arc polarized structures.

\subsection{Integration}

The material in this section may be bypassed without loss of continuity. We first observe that to obtain only the hodogram shape, but not the x-dependence, one can divide Eq. (1) by Eq. (2), thus eliminating the space variable $x$ and obtaining a first-order differential equation, which, together with Eq. (3), describes $B_{\mathrm{y}}$ as a function of $B_{\mathrm{z}}$. However, since $A_{\mathrm{x}}^{2}$ is expressed in Eq. (3) as a function of $B_{\mathrm{t}}^{2}$ and $B_{\mathrm{z}}$, a more convenient approach is to first combine Eqs. (1) and (2) to obtain

$\lambda_{i 2} \frac{A_{\mathrm{x}}^{2}}{A_{\mathrm{x} 2}} \frac{d B_{\mathrm{t}}^{2} / 2}{d x}=\left(A_{\mathrm{x} 2}^{2}-1\right) B_{\mathrm{z} 2} B_{\mathrm{y}}$

If Eq. (2) is now divided by Eq. (6), one obtains our basic hodogram equation

$2 B_{\mathrm{z} 2}\left(A_{\mathrm{x} 2}^{2}-1\right) \frac{d B_{\mathrm{z}}}{d B_{\mathrm{t}}^{2}}=\left(A_{\mathrm{x}}^{2}-1\right)$

where $A_{\mathrm{x}}^{2}$ is given by Eq. (3) as a function of $B_{\mathrm{t}}^{2}$ and $B_{\mathrm{z}}$.

Equation (7) can now be integrated analytically by use of the following variables in place of $B_{\mathrm{t}}^{2}$ and $B_{\mathrm{z}}$ :

$$
\begin{gathered}
R \equiv \frac{\gamma / 2}{\gamma+1}\left[2 A_{\mathrm{x} 2}^{2}+\frac{\beta_{2}}{\cos ^{2} \theta_{2}}+\left(1-\frac{B_{\mathrm{t}}^{2}}{B_{\mathrm{z} 2}^{2}}\right) \tan ^{2} \theta_{2}\right] \\
Z^{2} \equiv R^{2}+\frac{\gamma-1}{\gamma+1}\left(\tan ^{2} \theta_{2}\right)\left[\frac{B_{\mathrm{t}}^{2}}{B_{\mathrm{z} 2}^{2}}+2\left(A_{\mathrm{x} 2}^{2}-1\right) \frac{B_{\mathrm{z}}}{B_{\mathrm{z} 2}}\right. \\
\left.+\left(A_{\mathrm{x} 2}^{2}-1\right)^{2}-\frac{A_{\mathrm{x} 2}^{2}}{\sin ^{2} \theta_{2}}\left(A_{\mathrm{x} 2}^{2}+\frac{\gamma \beta_{2}}{\gamma-1}\right)\right]
\end{gathered}
$$

In this notation, the expression for $A_{\mathrm{x}}^{2}$ in Eq. (3) is simply $A_{\mathrm{x}}^{2}=R \pm Z$ and, after differentiation of Eqs. (8) and (9) with 
respect to $R$ and some straightforward algebra, the differential equation (7) becomes

$\gamma Z \frac{d Z}{d R}=R \mp(\gamma-1) Z$

This equation is seen to be homogeneous; it is therefore solvable by use of the new variable $U \equiv Z / R$ in place of $Z$. It then becomes separable and can be written as

$\frac{\gamma U d U}{1-\gamma U^{2} \mp(\gamma-1) U}=\frac{d R}{R}$

with solution

$(R \mp \gamma Z)\left((R \pm Z)^{\gamma}=C_{ \pm}\right.$

Assuming $Z$ to be the positive root of Eq. (9), the upper signs in Eq. (12) represent supersonic conditions and the lower signs, which are the relevant ones for our solar wind application, represent subsonic conditions. Given the definitions of $R$ and $Z$ in Eqs. (8) and (9), in which $B_{\mathrm{t}}^{2}=B_{\mathrm{y}}^{2}+B_{\mathrm{z}}^{2}$, we see that Eq. (12) provides a general relationship between $B_{\mathrm{y}}^{2}$ and $B_{\mathrm{Z}}$ that describes all possible hodogram trajectories around the fixed point 2 . Where they exist (i.e., where spiral points occur in the resistive shock model described by Hau and Sonnerup, 1990), non-dissipative wave trains around the fixed points 1,3 , or 4 are described by the same formulas but with the subscript 2 replaced by the subscript 1, 3, or 4 . Individual hodogram curves are defined by the value of the constant of integration $C_{ \pm}$. The fact that only $B_{\mathrm{y}}^{2}$ but not $B_{\mathrm{y}}$ appears shows that all these curves are symmetric about the $B_{\mathrm{Z}}$-axis.

Because Eq. (3) is of the form $A_{\mathrm{x}}^{2}=R \pm Z$, Eq. (12) can also be written as

$\left[(\gamma+1) R-\gamma A_{\mathrm{x}}^{2}\right] A_{\mathrm{x}}^{2 \gamma}=C_{ \pm}$

From this expression, one sees that the hodogram is expressed in parametric form: a chosen value of the parameter $A_{\mathrm{x}}^{2}$ allows calculation of $R$ from Eq. (13) and then $Z$ from $A_{\mathrm{x}}^{2}=R \pm Z$ (for the subsonic case, the negative sign must be used). The value of $R$ then allows $B_{\mathrm{t}}^{2}$ to be obtained from Eq. (8), whereupon $B_{\mathrm{Z}}$ can be found from Eq. (9).

The value of the constant of integration, $C_{ \pm}$, is obtained, for example by putting the turning point conditions, $A_{\mathrm{x}}^{2}=1$ and $R=R^{*}$, into Eq. (13) to give

$C_{ \pm}=\left[(\gamma+1) R^{*}-\gamma\right]$

The value for $R^{*}$ is obtained by first specifying $B_{\mathrm{z}}^{*} / B_{\mathrm{t} 2}$, the non-dimensional value of $B_{\mathrm{Z}}$ at the turning points, and then finding the corresponding value of $B_{\mathrm{t}}^{* 2} / B_{\mathrm{z} 2}^{2}$ from Eq. (4). The latter value is then substituted into Eq. (8) to give $R^{*}$.

Once the hodogram has been determined, as described above, we know the functions $B_{\mathrm{y}}\left(A_{\mathrm{x}}^{2}\right)$ and $B_{\mathrm{z}}\left(A_{\mathrm{x}}^{2}\right)$. The relationships describing the $\mathrm{x}$-dependence of the field can then be obtained in the form of quadratures by integration of Eq. (1) or Eq. (2) to give expressions in the form of either of the following two integrals

$\frac{x}{\lambda_{i 2} A_{\mathrm{x} 2}^{-1}}=\int \frac{A_{\mathrm{x}}^{2}}{A_{\mathrm{x}}^{2}-1} \frac{d B_{\mathrm{z}}}{B_{\mathrm{y}}}=\int \frac{A_{\mathrm{x}}^{2} d B_{\mathrm{y}}}{B_{\mathrm{z} 2}\left(A_{\mathrm{x} 2}^{2}-1\right)-B_{\mathrm{z}}\left(A_{\mathrm{x}}^{2}-1\right)}$

where, as before, $A_{\mathrm{x}}^{2}=R \pm Z$. As already mentioned, these expressions show that structures much wider than the ion inertial length $\lambda_{i 2}$ can occur, but only when $A_{\mathrm{x}}^{2}$ remains very close to unity at all points on the hodogram curve; this happens only when $A_{\mathrm{x} 2}^{2}$ is very close to unity. We note again that $A_{\mathrm{x} 2}$ is positive when $v_{\mathrm{x}}$ and $B_{\mathrm{x}}$ have the same sign and is negative when they have opposite signs. Starting at the left turning point in Fig. $2 \mathrm{a}$ or Fig. 3, and assuming $A_{\mathrm{x} 2}$ to be positive, one then sees the inner (lower) branch first, followed by the outer (upper) branch. When $A_{\mathrm{x} 2}$ is negative, the order, and therefore the overall sense of motion around of the banana-shaped hodogram, is reversed.

The integrand of the first integral in Eq. (15) is singular at the left and right turning points for $B_{\mathrm{Z}}$, where $A_{\mathrm{x}}^{2}=1$, and also at the top of the hodogram branches (the symmetry points) where $B_{\mathrm{y}}=0$. In the second integral, there is a singularity at the turning points for $B_{\mathrm{y}}$ instead. These singularities are all integrable.

\subsection{Numerical examples}

To illustrate the details of the solution, we select the following parameters: $\gamma=2 ; \beta_{2}=1 ; A_{\mathrm{x} 2}^{2}=1.001 ; \cos ^{2} \theta_{2}=0.004$ $\left(\theta_{2}=86.4^{\circ}\right) ; B_{\mathrm{z}}^{*}=0$. We will give certain results with high accuracy so that they can be used for code validation. From Eq. (4), we find $\left(B_{\mathrm{t}}^{*} / B_{\mathrm{z} 2}\right)^{2}-1=-4.00000401606 \times 10^{-3}$ and, from Eq. (8), $R^{*}=84.332667$. With this $R^{*}$ value, Eq. (14) gives the constant of integration $C_{ \pm}=250.998001$. Equation (13) now gives a relationship between $R$ and the parameter $A_{\mathrm{x}}^{2}$. For each choice of the latter, we can use the resulting $\mathrm{R}$-value in $\mathrm{Eq}$. (8) to calculate the corresponding value of $\left(B_{\mathrm{t}}^{*} / B_{\mathrm{z} 2}\right)^{2}-1$. Using $Z^{2}=\left(A_{\mathrm{x}}^{2}-R\right)^{2}$, we can then find $Z^{2}$ and finally use Eq. (9) to get $B_{\mathrm{z}} / B_{\mathrm{z} 2}$. By making a set of choices of $A_{\mathrm{x}}^{2}$ slightly larger (smaller) than unity, we can thus map out the outer (inner) branch of the hodogram. Note that the choices of $A_{\mathrm{x}}^{2}$ are restricted by the requirement $B_{\mathrm{t}}^{2} \geq B_{\mathrm{z}}^{2}$; the equality applies at the symmetry point, at the top of each branch, where $B_{\mathrm{y}}=0$. These two points must be found by trial and error. The result for our numerical example is:

Top of outer branch:

$A_{\mathrm{x}}^{2}=1.0477398 ; \quad B_{\mathrm{z}} / B_{\mathrm{z} 2}=1.04181769$

Top of inner branch:

$A_{\mathrm{x}}^{2}=0.9582725 ; \quad B_{\mathrm{z}} / B_{\mathrm{z} 2}=0.95217432$

The resulting hodogram is shown as the blue doublebranched curve in Fig. 3a, which curve corresponds to the 


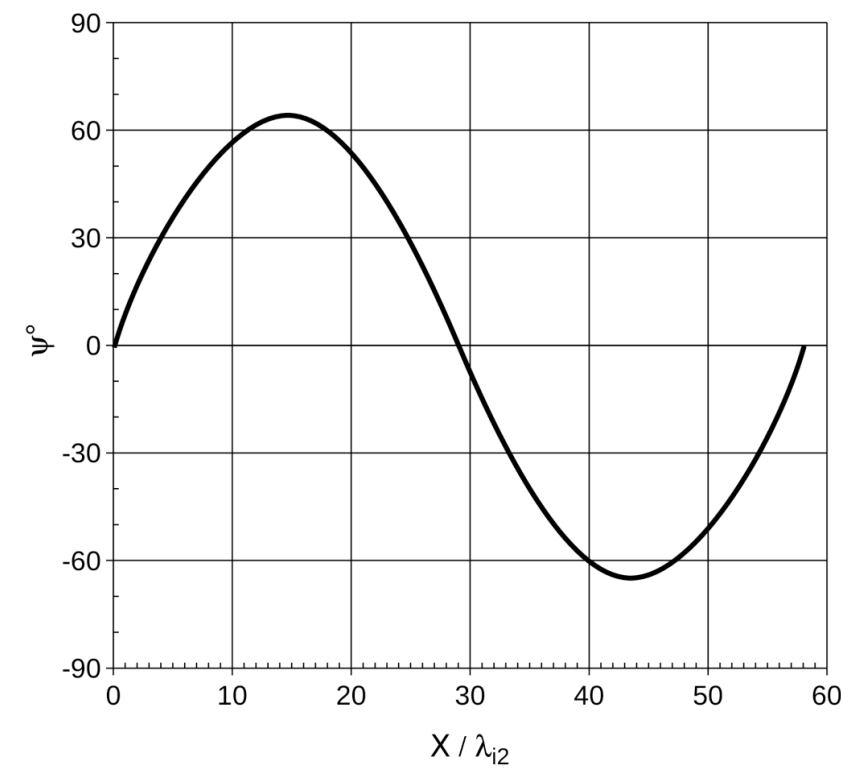

Fig. 4. Plot of the angle $\psi=\tan ^{-1}\left(B_{\mathrm{y}} / B_{\mathrm{z}}\right)$ of the tangential field relative to the vertical axis of the hodogram (see Fig. 2a) versus distance $x$. Parameters are the same as for the hodogram curve in Fig. 3b.

choice, $B_{\mathrm{z}}^{*} / B_{\mathrm{z} 2}=0$, made above. The tangential field tips back and forth in the angle range $-90^{\circ} \leq \psi \leq+90^{\circ}$, where $\psi=\tan ^{-1}\left(B_{\mathrm{y}} / B_{\mathrm{z}}\right)$. When $A_{\mathrm{x} 2}$ is positive, the sense of the field rotation on the inner branch is toward the right in the figure (from negative towards positive angles $\psi$ ), followed by the return rotation (to the left) along the outer branch. When $A_{\mathrm{x} 2}$ is negative, the sense is reversed: rotation on the inner branch is to the left and on the outer branch to the right. Similarly, the black double-branched curve corresponds to $B_{\mathrm{z}}^{*} / B_{\mathrm{z} 2}=+0.5$ and an angle range of approximately $-60^{\circ} \leq$ $\psi \leq+60^{\circ}$. The two branches of the red hodogram curve are close to the two separatrices, which intersect at the fixed point 3 (a saddle point) and form the boundaries of the domain in the hodogram plane within which the solutions of interest to us are located. The separatrices themselves describe two solitary waves of opposite polarization, each showing a field rotation of $360^{\circ}$.

The results in expressions (16) and (17) can be used in Eq. (1) to calculate the spatial derivative $\left(\lambda_{i 2} / B_{\mathrm{z} 2}\right) d B_{\mathrm{y}} / d x$ on top of the two branches, where $B_{\mathrm{y}}=0$. Its value is -0.04654 for the outer branch and +0.04253 for the inner branch (assuming $A_{\mathrm{x} 1}$ is positive). The two signs are opposite as expected, but contrary to the observed behavior, the corresponding two angular rotation rates $|d \psi / d x|$ are nearly equal.

A hodogram for parameter values applicable to the event in Fig. 1 is shown in Fig. 3b, with a plot of the corresponding tilt angle $\psi$ of the tangential field versus distance $x$ along the normal direction in Fig. 4. In this latter plot, the beginning point of the curve, at $x=0 ; \psi=0$, corresponds to the top of the inner branch of the hodogram. The field then tips to the right until the right turning point at $\psi=+65^{\circ}$ is reached and a transition to the outer branch occurs. Along the latter, the angle then decreases until the left turning point at $\psi=-65^{\circ}$ is reached. There, a transition to the inner branch occurs and the angle starts increasing until $\psi=0$ is reached again at the top of the inner branch. The curve does not display any substantial differences between the field rotation rates on the inner and outer branches and we have been unable to find parameter values for which such a difference occurs. The solution shown has been matched to the observed thickness of the inner branch (in the range $25 \lambda_{i 2}$ to $32 \lambda_{i 2}$ ) and the observed branch separation $(\sim 13 \%)$, which necessitated replacing the isentropic model $(\gamma=5 / 3)$ by a polytropic one, with the nearly isothermal choice $\gamma=1.134$.

\section{Cluster event}

We now analyze in more detail the structure in Fig. 1, seen by Cluster $1(\mathrm{C} 1)$. We will examine the normal magnetic field and flow, the Walén relation, and the sense of flow of the 'strahl' electrons relative to the sense of the magnetic field. We will also calculate spatial dimensions of the fast and slow rotations and make various comparisons with our theoretical model. Finally, we will summarize the results from all four Cluster spacecraft. Note that all our MVAB calculations are based on the full resolution magnetometer data (22.4 samples/s). However, calculations that include plasma information are limited to the $4 \mathrm{~s}$ resolution of the CIS/HIA instrument.

The eigenvectors from MVAB, used as axes in Fig. 1, form a right-handed orthonormal triad, $\left(\hat{\boldsymbol{x}}_{1} ; \hat{\boldsymbol{x}}_{2} ; \hat{\boldsymbol{x}}_{3}\right)$, with the positive minimum-variance axis, $\hat{\boldsymbol{x}}_{1}$, pointing towards the sun (this convention is maintained throughout our paper). This minimum-variance axis provides a single-spacecraft estimate of the normal vector $\boldsymbol{n}$, which is therefore always sunward directed, according to our convention. The fact that the average field component in this direction is negative (see the upper right hodogram projection in Fig. 1) means that, with this estimate of $\boldsymbol{n}$, the normal field component points away from the sun. However, the intermediate and smallest eigenvalues from MVAB have the relatively low ratio $\lambda_{2} / \lambda_{1}=1.41 / 0.26=5.4$, indicating that the normal direction may not be particularly well determined. The reason for the poor ratio is that the field fluctuations in the normal direction during the slow rotation increase the corresponding variance, $\lambda_{1}$. It may also corrupt the MVAB estimate of the orientation of $\boldsymbol{n}$. For this reason, we obtain the normal direction by instead applying MVAB to only the short data interval containing the rapid field rotation. The resulting hodogram set is shown in Fig. 5, with numerical information in Table 1. One can see that the eigenvalue ratio now has increased to $\lambda_{2} / \lambda_{1}=230$, indicating that the minimum variance direction should provide an accurate estimate of the true 
Table 1. Results from MVAB and HT/Walén analysis of Cluster and Ulysses events.

\section{CLUSTER 1 2003-02-03}

MVAB: 19:11:32.000-19:11:36.995 UT Resolution: 0.0446 s

Eigenvectors $\hat{\boldsymbol{x}}_{i}$ GSE $(i=1 ; 2 ; 3)$

$(+0.924 ;+0.106:+0.368)=n$

$(+0.233 ;+0.608-0.759)$

$(-0.304 ;+0.787 ;+0.537)$

HT/Walén: 19:11:10-19:14:34 UT

Walén slope $=+0.89 c c_{\text {Walén }}=+0.991$
Eigenvalues $\lambda_{i} \mathrm{nT}^{2}$

0.00657

1.509

31.44

Plasma resolution: $4 \mathrm{~s}$

Strahl pitch angle $=180^{\circ}$

\begin{tabular}{|c|c|c|}
\hline \multicolumn{3}{|c|}{ CLUSTER 2 2003-02-03 } \\
\hline MVAB: 19:11:27.032-19:11:30.956 UT & \multicolumn{2}{|c|}{ Resolution; $0.0446 \mathrm{~s}$} \\
\hline Eigenvectors $\hat{\boldsymbol{x}}_{i}$ GSE $(i=1 ; 2 ; 3)$ & Eigenvalues $\lambda_{i}(\mathrm{nT})^{2}$ & $\langle\boldsymbol{B}\rangle \cdot \boldsymbol{n}(\mathrm{nT})$ \\
\hline$(+0.943 ;+0.333 ;-0.009)=n$ & 0.00891 & $-1.58 \pm 0.07^{\mathrm{a}}(-2.16)^{\mathrm{b}}$ \\
\hline$(-0.166 ;+0.447 ;-0.879)$ & 0.812 & \\
\hline$(-0.288 ;+0.830 ;+0.477)$ & 26.2 & \\
\hline \multicolumn{3}{|c|}{ CLUSTER 3 2003-02-03 } \\
\hline MVAB: 19:11:27.006-19:11:31.957 UT & \multicolumn{2}{|c|}{ Resolution: $0.0446 \mathrm{~s}$} \\
\hline Eigenvectors $\hat{\boldsymbol{x}}_{i}$ GSE $(i=1 ; 2 ; 3)$ & Eigenvalues $\lambda_{i}(\mathrm{nT})^{2}$ & $\langle\boldsymbol{B}\rangle \cdot \boldsymbol{n}(\mathrm{nT})$ \\
\hline$(+0.881 ;+0.358:+0.311)=n$ & 0.0491 & $+0.31 \pm 0.08^{\mathrm{a}}(+0.40)^{\mathrm{b}}$ \\
\hline$(+0.043 ;+0.592-0.805)$ & 2.15 & \\
\hline$(-0.472 ;+0.722 ;+0.506)$ & 33.2 & \\
\hline HT/Walén: 19:11:10-19:14:34 UT & Plasma resolution: $4 \mathrm{~s}$ & \\
\hline \multirow{2}{*}{\multicolumn{3}{|c|}{$\begin{array}{l}\mathrm{GSE}(\mathrm{km} / \mathrm{s}): \boldsymbol{V}_{\mathrm{HT}}=(-505.7 ;+44.6 ;-20.1)\langle\boldsymbol{v}\rangle=(-512.4 ; 27.0 ; 24.8)\left(\langle\boldsymbol{v}\rangle-\boldsymbol{V}_{\mathrm{HT}}\right) \cdot \boldsymbol{n}=+1.8 \\
\text { Walén slope }=+0.79 c c_{\text {Walén }}=+0.995 \quad \text { Strahl pitch angle }=180^{\circ}\end{array}$}} \\
\hline & & \\
\hline \multicolumn{3}{|c|}{ CLUSTER 4 2003-02-03 } \\
\hline MVAB: 19:11:24.048-19:11:28.463 UT & \multicolumn{2}{|c|}{ Resolution; $0.0446 \mathrm{~s}$} \\
\hline Eigenvectors $\hat{\boldsymbol{x}}_{i}$ GSE $(i=1 ; 2 ; 3)$ & Eigenvalues $\lambda_{i}(\mathrm{nT})^{2}$ & $\langle\boldsymbol{B}\rangle \cdot \boldsymbol{n}(\mathrm{nT})$ \\
\hline$(+0.842 ;+0.497:-0.211)=n$ & 0.0390 & $-1.56 \pm 0.08^{\mathrm{a}}(-3.01)^{\mathrm{b}}$ \\
\hline$(-0.389 ;+0.289-0.875)$ & 1.47 & \\
\hline$(-0.374 ;+0.819 ;+0.436)$ & 28.8 & Strahl pitch angle $=180^{\circ}$ \\
\hline \multicolumn{3}{|c|}{ ULYSSES 1995-07-29 } \\
\hline MVAB: 23:40:47.710-23:43:19.720 UT & \multicolumn{2}{|c|}{ Resolution: $1 \mathrm{~s}$} \\
\hline Eigenvectors $\hat{\boldsymbol{x}}_{i}$ RTN $(i=1 ; 2 ; 3)$ & Eigenvalues $\lambda_{i}(\mathrm{nT})^{2}$ & $\langle B\rangle \cdot n(\mathrm{nT})$ \\
\hline$(-0.853 ;-0.092:+0.513)=n$ & 0.00903 & $-0.42 \pm 0.02^{\mathrm{a}}(-0.68)^{\mathrm{b}}$ \\
\hline$(-0.447 ;-0.378 ;-0.811)$ & 0.263 & \\
\hline$(+0.269 ;-0.921 ;+0.282)$ & 1.28 & \\
\hline HT/Walén: 23:25:30.700-23:43:18.720 & Plasma resolutic & n: 242 s. (4 points) \\
\hline $\begin{array}{l}\text { RTN }(\mathrm{km} / \mathrm{s}): V_{\mathrm{HT}}=(+800.0 ;-13.4 ;+1.5 \\
\text { Walén slope }=-0.607 c c_{\text {Walén }}=-0.982\end{array}$ & $\langle\boldsymbol{v}\rangle=(+777.8 ;-9.6 ;-9$ & 9) $\left(\langle\boldsymbol{v}\rangle-\boldsymbol{V}_{\mathrm{HT}}\right) \cdot \boldsymbol{n}=+12.7$ \\
\hline
\end{tabular}

a Statistical errors only, estimated from Eq. (8.24) in Sonnerup and Scheible (1998).

b Value in parentheses uses same normal vector but $\langle\boldsymbol{B}\rangle$ from full event.

normal direction. Figure 5 suggests that a nearly perfect planar structure (in which $\boldsymbol{B} \cdot \boldsymbol{n}$ would be strictly constant) may have been sampled. Along this new normal direction, which forms an angle of $14^{\circ}$ with the direction derived from the total event (see Fig. 1), the normal field component is now positive, not only during the fast rotation $(\langle\boldsymbol{B}\rangle \cdot \boldsymbol{n}=+0.53 \mathrm{nT}$, where $\langle\ldots\rangle$ denotes an average over the data set), as shown in the upper right hodogram projection in Fig. 5, but, with the exception of a few data points, also during the combined fast and slow rotations $(\langle\boldsymbol{B}\rangle \cdot \boldsymbol{n}=+0.68 \mathrm{nT})$. The purely statistical uncertainty of these normal components, calculated from Eqs. (8.23) and (8.24) in the review of MVAB by Sonnerup and Scheible (1998), is only about $\pm 0.04 \mathrm{nT}$. It is caused mainly by the uncertainty in the normal vector orientation under rotation about the maximum variance axis and its small value is the result of the high time resolution used. Experiments using MVAB with five lower sampling rates give results for $\langle\boldsymbol{B}\rangle \cdot \boldsymbol{n}$ that all fall within this small error interval. In 


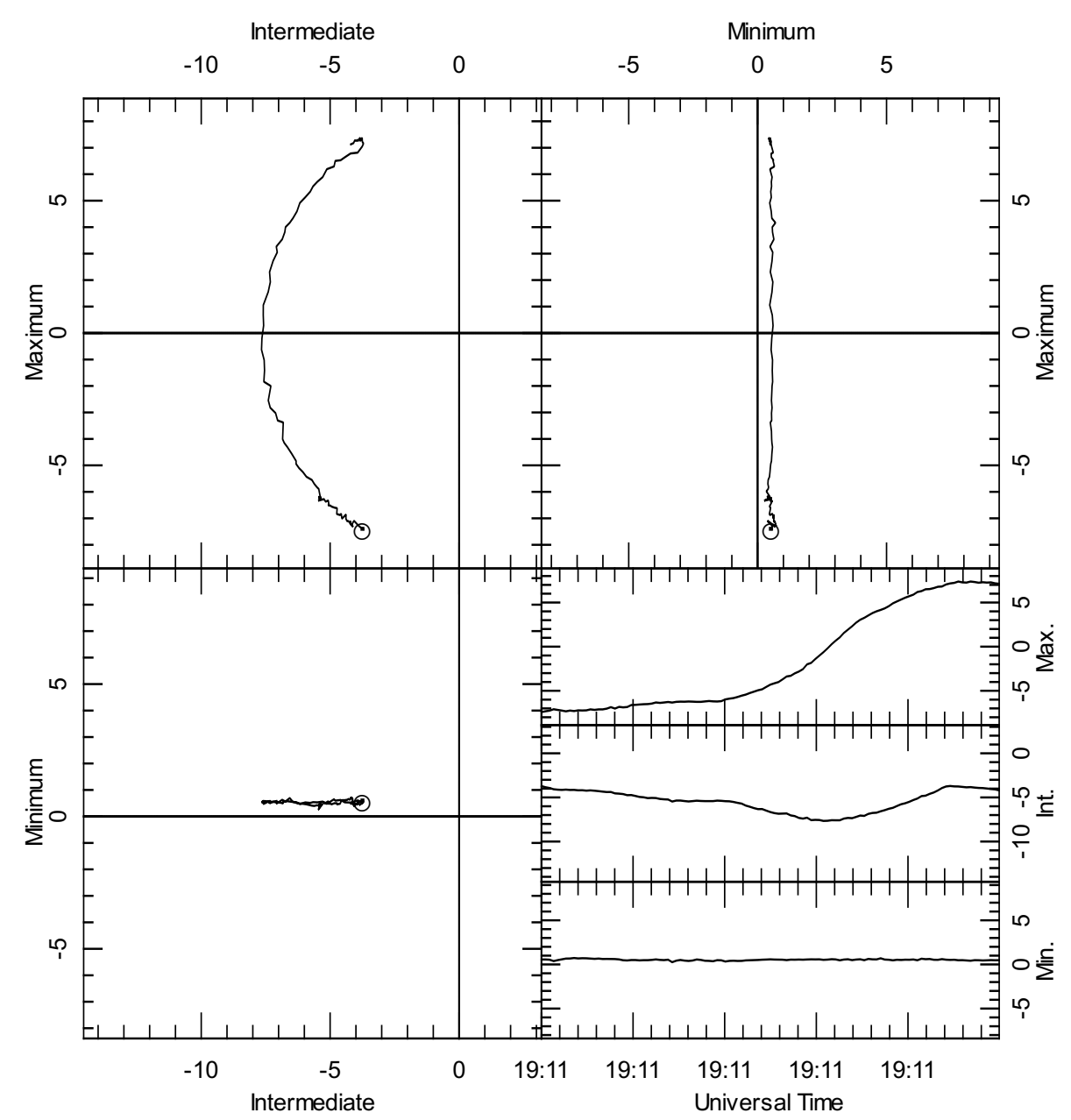

Time Interval (UT): 2003-02-03 19:11:32.000 - 19:11:36.995

Fig. 5. Cluster 1 event in Fig. 1: Hodogram for the rapid field rotation alone. Resolution is 22.4 samples/s. Time axis in lower right panel spans $5 \mathrm{~s}$ from 19:11:32 to 19:11:37. The eigenvalue ratio is $\lambda_{2} / \lambda_{1}=230$; further details from the MVAB calculation are given in Table 1 .

experiments with nested larger and smaller data intervals (in the range $-3 \mathrm{~s}$ to $+5 \mathrm{~s}$ ), the variations are larger but $\langle\boldsymbol{B}\rangle \cdot \boldsymbol{n}$ remains positive. Calibration errors, estimated not to exceed $0.1 \mathrm{nT}$ for the data set used here, is an additional source of uncertainty in $\langle\boldsymbol{B}\rangle \cdot \boldsymbol{n}$. But it is still much too small to call into question the positive sign of $\langle\boldsymbol{B}\rangle \cdot \boldsymbol{n}$.

The theoretical hodogram in Fig. $3 b$ is based on the parameter values for the Cluster event. If it is rotated counterclockwise so as to assume the same orientation as the tangential hodogram in Fig. 1 (or Fig. 5), then the sense of motion along the hodogram trajectory, from bottom to top along the inner branch, followed by motion from top to bottom on the outer branch, is seen to be the same in the two figures. But the sense of motion indicated in Fig. $3 b$ is based on the assumption that $A_{\mathrm{x} 1}$ is positive, i.e., that the normal flow and the normal field have the same sign. Since the av- erage normal field, calculated above, is positive, our model predicts that the normal flow should also be positive and that, more generally, the Walén slope for the entire event should be positive and near +1 , since the flow is nearly Alfvénic in the model. The Walén slope is the slope of the regression line in a scatter plot of the flow velocity components in the deHoffmann-Teller (HT) frame versus the corresponding components of the Alfvén velocity. The HT frame (see the review by Khrabrov and Sonnerup, 1998) is used as our prediction of the proper frame of the structure. As recorded in Table 1, the regression line slope is indeed positive and equal to +0.89 , with a correlation coefficient +0.991 . The Alfvén velocities used in the Walén test are based on the assumption that all measured particles are protons and that the pressure is isotropic. If $8.8 \%$ of them were in fact alpha particles, the slope would increase to +1 . However, measurements by the 
ACE spacecraft during the event indicate an alpha to proton ratio of only $4 \%$. Therefore, pressure anisotropy and/or other factors must also play a role.

The Alfvén speed based on the normal field component of $\langle\boldsymbol{B}\rangle \cdot \boldsymbol{n}=+0.53 \mathrm{nT}$ (for the rapid rotation only) and a number density (assuming protons only) of $n=9.6 \mathrm{~cm}^{-3}$ is $V_{\mathrm{Ax}}=+3.7 \mathrm{~km} / \mathrm{s}$. Using the same normal vector but the average normal field, $\langle\boldsymbol{B}\rangle \cdot \boldsymbol{n}=+0.68 \mathrm{nT}$, for the entire structure (fast and slow rotation together), the corresponding result is $V_{\mathrm{Ax}}=+4.8 \mathrm{~km} / \mathrm{s}$. Since the Walén slope is positive as predicted by our theory, the plasma flow speed across the structure should also be positive, i.e., it should be directed toward the sun, and be of comparable magnitude (on account of the Walén slope it should be some $11 \%$ below the range $3.7-4.8 \mathrm{~km} / \mathrm{s}$ ). In other words, we infer that the structure was propagating anti-sunward through the ambient plasma at a speed in, or near, this range. To accurately measure such a small plasma flow relative to the structure is probably beyond the limit of the combined accuracy of the CIS/HIA instrument and of the determination of the proper frame velocity (the HT velocity). The relative velocity is the difference between two speeds of the order of $500 \mathrm{~km} / \mathrm{s}$, which means that the accuracy of each must be $0.5 \%$ or better. These reservations notwithstanding, we have performed the calculation. Using the average measured plasma velocity $\langle\boldsymbol{v}\rangle$ and the frame velocity $\boldsymbol{V}_{\mathrm{HT}}$, both based on the data in the time interval of the entire event, together with the $\boldsymbol{n}$ vector from the rapid rotation only, we find a relative plasma velocity of $\left(\langle\boldsymbol{v}\rangle-\boldsymbol{V}_{\mathrm{HT}}\right) \cdot \boldsymbol{n}=+7.1 \mathrm{~km} / \mathrm{s}$. Within the large uncertainties, this result can be considered consistent with the estimates of the normal component of the Alfvern velocity given above. The overall consistency with the model indicates that at least the signs of these two velocity components have been correctly obtained.

The time durations of the rapid and slow rotations in Fig. 1 are about $4-5 \mathrm{~s}$ and $170 \mathrm{~s}$, respectively. By use of the HT frame velocity $\boldsymbol{V}_{\mathrm{HT}}=(-511.8 ;+44.4 ;-12.8) \mathrm{km} / \mathrm{s}$ and the normal vector $\boldsymbol{n}=(0.924 ; 0.106 ; 0.368)$ from Table 1 , the corresponding widths of the structure are $1880-2350 \mathrm{~km}$ and $80390 \mathrm{~km}$, respectively. Noting that the ion inertial length, with an average density of 9.6 protons $/ \mathrm{cm}^{3}$, is $\lambda_{i}=73.6 \mathrm{~km}$, we then find the rapid rotation to occur over a distance along the normal that is in the range $26-32 \lambda_{i}$; the corresponding distance for the slow rotation is some 43-34 times larger. The result for the inner-branch width may be compared with the spatial scale of $29 \lambda_{i}$ in Fig. 4. As already mentioned, the values $\beta_{2}, \theta_{2}$, and $\Delta \psi$, used to generate the figure, correspond to the observed values and the pair of values $A_{\mathrm{x} 2}^{2}=1.0063$ and $\gamma=1.134$ was chosen so that the theoretical hodogram would reproduce the observed gap between the inner and outer branch of the hodogram (compare Figs. 1 and $3 b$ ) and at the same time give a spatial scale of about $29 \lambda_{i}$ for the rapid rotation. It appears that the polytropic version of the model is capable of reproducing the hodogram as well as the spatial scale of its inner branch.
As expected, the PEACE instrument onboard Cluster 1 saw the beam of electrons, referred to as "strahl". By examining the electron spectrograms, available on the PEACE web site, at $0^{\circ}$ and $180^{\circ}$ pitch angle, the "strahl" was found to be directed anti-parallel to the magnetic field (peak flux at $180^{\circ}$ pitch angle). These electrons are believed to originate on the sun. Because the normal magnetic-field component points sunward in our discontinuity, this means the "strahl" electrons were crossing the structure from its sunward to its earthward side. This behavior is consistent with the field lines on the sunward side being "rooted" in the sun and the electrons accessing the earthward side by flowing across the structure.

Overall for $\mathrm{C} 1$, there is good consistency between the observations and the behavior predicted by the theoretical model. The major disagreement is that the model fails to predict the slowness of the field rotation on the outer hodogram branch. In this context, it is noted that the model does not include the possible net effect of the field fluctuations.

The other three Cluster spacecraft recorded tangential hodograms similar to that shown for $\mathrm{C} 1$ in Fig. 1, including the same sense of rotation of the field vector around the inner and outer hodogram branches. But there were some important differences. The normal vectors (as for $\mathrm{C} 1$, based on data from the fast-rotation interval only) were again very well determined but varied significantly from spacecraft to spacecraft (see Table 1 and Fig. 6). The sign of the normal field component and of the normal flow across the structure was positive and the same for $\mathrm{C} 3$ as for $\mathrm{C} 1$, while for $\mathrm{C} 2$ and $\mathrm{C} 4$ the normal field component had the reverse sign (see Table 1). The prediction from our theory is that the flow direction should then have reversed as well. Since the CIS instrument is not functional on $\mathrm{C} 2$, the actual direction of the plasma flow across the discontinuity could not be checked for that spacecraft. For C4, the HIA part of the CIS instrument is also not functional but the CODIF part is delivering data. These data, while less accurate and containing gaps, do in fact produce results that are consistent with the prediction $\left(\left(\langle\boldsymbol{v}\rangle-\boldsymbol{V}_{\mathrm{HT}}\right) \cdot \boldsymbol{n}=-19 \mathrm{~km} / \mathrm{s}\right.$; Walén slope $\left.=+0.5\right)$, at least in terms of signs. The implication is that the structures observed by $\mathrm{C} 2$ and $\mathrm{C} 4$ had the reverse propagation direction, heading sunward rather than earthward relative to the plasma, and, contrary to the case for $\mathrm{C} 1$ and $\mathrm{C} 3$, therefore having the slow field rotation on its upstream rather than its downstream side. Here the terms upstream and downstream refer to the plasma velocities as viewed in the HT frame. In the spacecraft frame of reference, the rapid rotation preceded the slow rotation for all four spacecraft, as shown in the time plot in Fig. 7. In this figure one can also see that the time order of the traversals was $\mathrm{C} 4, \mathrm{C} 2, \mathrm{C} 3$, and $\mathrm{C} 1$, with less than $1 \mathrm{~s}$ separation between the $\mathrm{C} 2$ and $\mathrm{C} 3$ crossings. In spite of this near simultaneity, there are noteworthy differences, in particular in the behavior of the GSE $B_{\mathrm{x}}$ component of the field seen by $\mathrm{C} 2$ and $\mathrm{C} 3$ (see the second panel in Fig. 7). Together with the large differences in the predicted normal direction, and the 


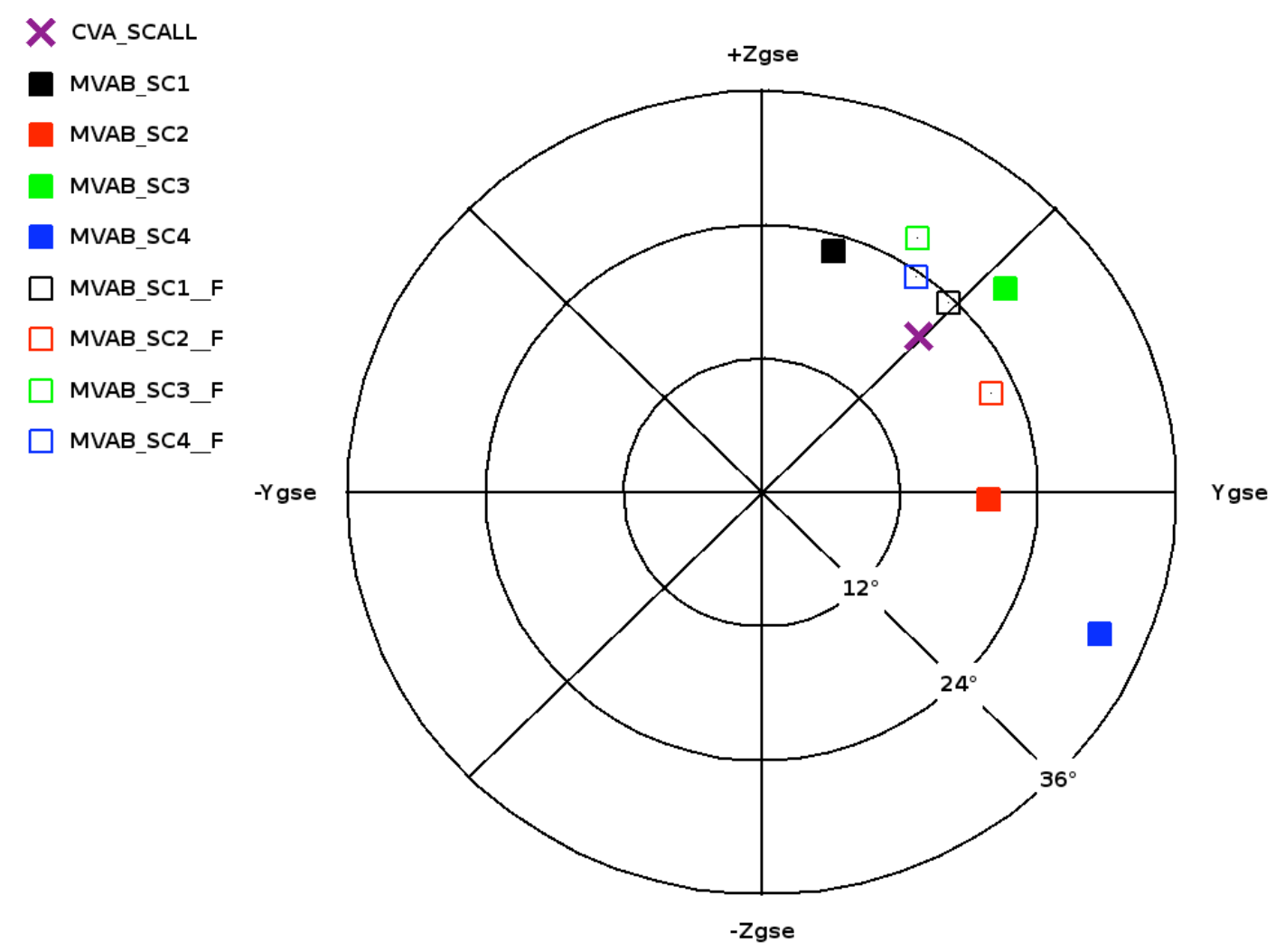

Fig. 6. Polar plot of MVAB normal vectors for the rapid rotation interval (filled squares) and for the full $(F=$ rapid + slow) time interval (unfilled squares) of the Cluster event (colors: C1 black; C2 red; C3 green; C4 blue). Time intervals are listed in Table 1. At the center is the direction of the GSE X-axis. Four-spacecraft timing, along with the constant velocity assumption, CVA (purple cross), gives $\boldsymbol{n}_{\mathrm{CVA}}=$ $(0.9436 ; 0.2306 ; 0.2374)$, using the following spacecraft locations (GSE components in $R_{\mathrm{E}}$ ) and time lags (Fig. 7) relative to C4:

$\begin{array}{rlr}\mathrm{C} 1 & =\left[\begin{array}{llr}16.8374 & 8.3899 & d-1.7107\end{array}\right], & +9.098 \mathrm{~s} \\ \mathrm{C} 2 & =\left[\begin{array}{llr}17.2566 & 8.2638 & -1.4753\end{array}\right], & +3.077 \mathrm{~s} \\ C 3 & =\left[\begin{array}{llr}17.2965 & 8.3549 & -1.9919\end{array}\right], & +3.969 \mathrm{~s} \\ C 4 & =\left[\begin{array}{lll}17.3747 & 8.7685 & -1.5073\end{array}\right], & 0.000 \mathrm{~s}\end{array}$

reversal of the sign of the normal field component, such behavior indicates the presence of significant and unexplained 2-D or 3-D structure on scales of the spacecraft separation. Adding to the puzzle is the fact that the normal directions, determined by use of MVAB in the data interval of the rapid rotations, indicate that the four individual normal-vector determinations had very high quality, which in turn strongly suggests, locally 1-D structure during the fast field rotation at each of the spacecraft.

Because the time lag between the four crossings is so small, one expects that the "strahl" electrons were flowing anti-parallel to the field and that the Walén slope was positive at and around all four crossings. For $\mathrm{C} 3$ and $\mathrm{C} 4$, these features were directly confirmed by CIS/HIA, CIS CODIF, and PEACE . No PEACE or CIS data were available during the $\mathrm{C} 2$ encounter but we infer with confidence that the 'strahl' must have been at $180^{\circ}$ for this crossing too and that the Walén slope must have been positive.

\section{Ulysses event}

This event was observed by the Ulysses spacecraft on 29 July 1995, in the time interval 23:15 $30-23: 4320$ UT, at $80.2^{\circ}$ northern heliographic latitude and a distance of $2.0 \mathrm{AU}$. The event was first reported and discussed by Tsurutani et al. (1997) and has subsequently been further discussed in the literature (e.g., Tsurutani and Ho, 1999; Horbury and Tsurutani, 2001). It has the remarkable property that the spacecraft observed the slow rotation first, followed by the rapid rotation. Here we re-examine the event in the context of our theory. The hodogram representation of the magnetic field is shown in Fig. 8 for the entire event and in Fig. 9 for the rapid rotation only. The tangential field is seen to rotate by approximately $180^{\circ}$. In the tangential hodogram of Fig. 8, the inner (rapid) branch of the tangential hodogram is obscured by fluctuations on the outer (slow) branch but a plot of field magnitude versus time reveals a noticeably 


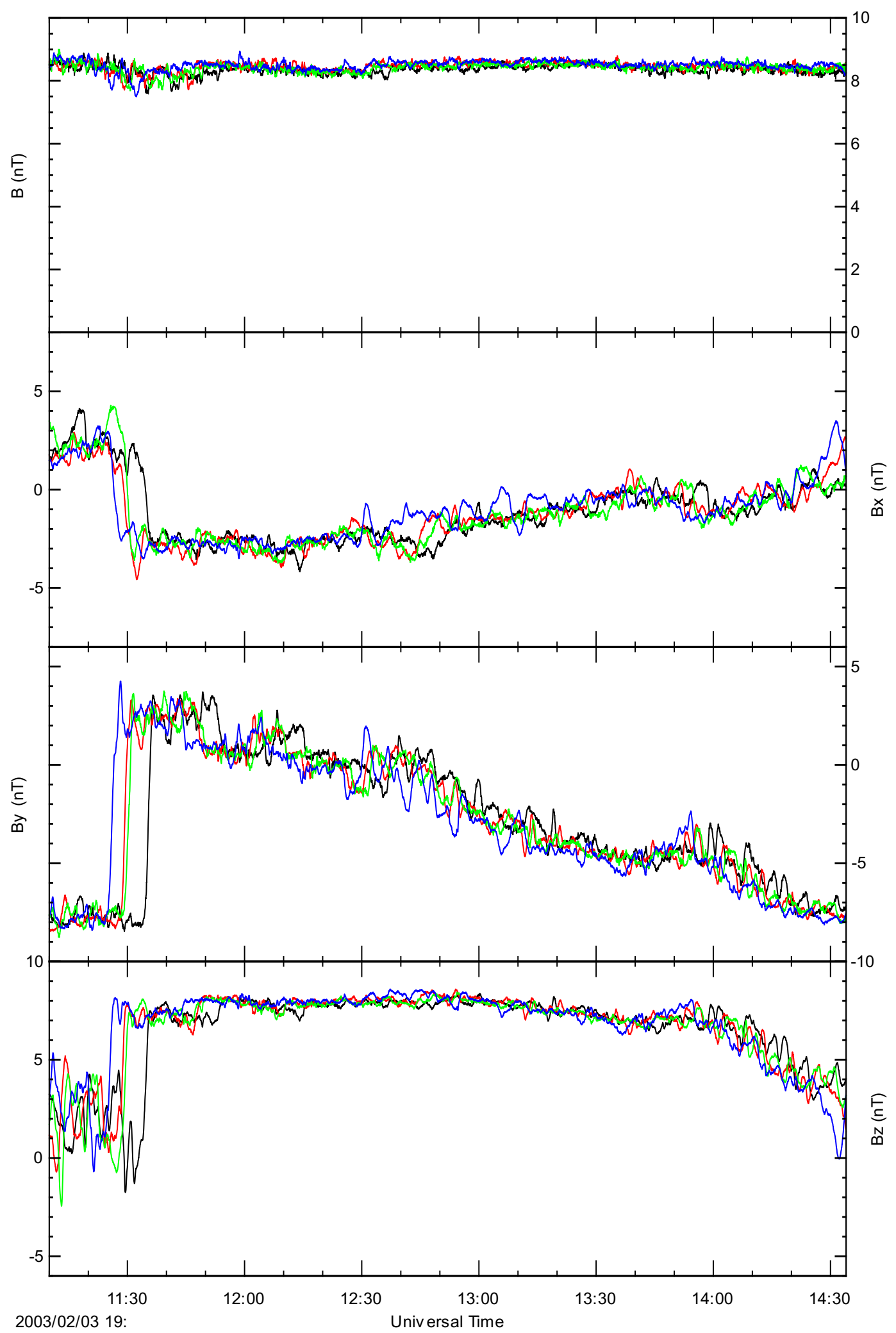

Fig. 7. Cluster event on 3 February 2003: Time plots of GSE magnetic field components, measured by each of the four spacecraft (C1 black; $\mathrm{C} 2$ red; $\mathrm{C} 3$ green; $\mathrm{C} 4$ blue). Resolution is 22.4 samples/s. 


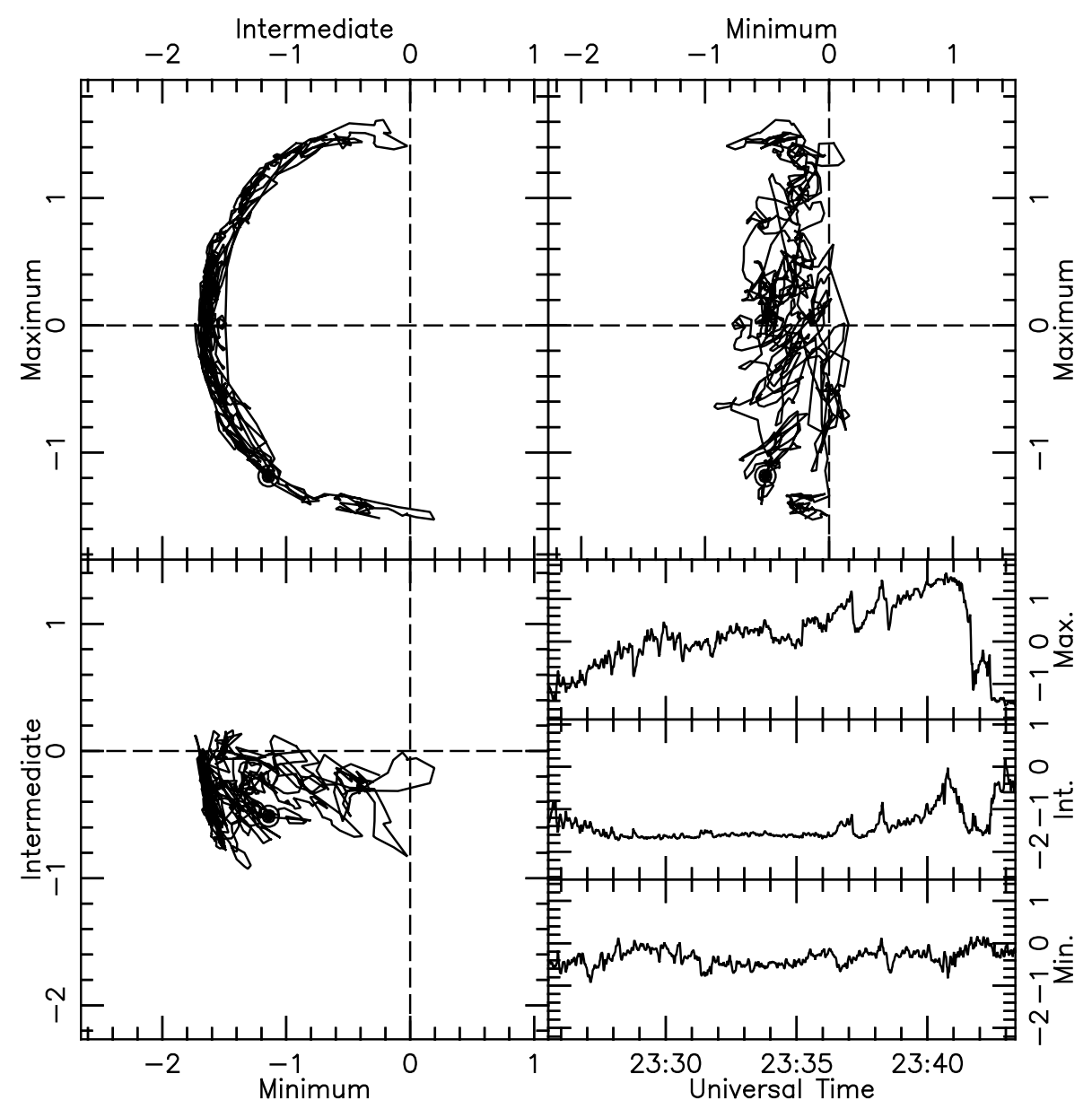

Time Interval (UT): 1995-07-29 23:25:30.700 - 23:43:21.720

Fig. 8. Variance results for Ulysses event seen at $2.0 \mathrm{AU}$ and $80.2^{\circ}$ northern heliographic latitude on 29 July 1995 . The event was first discussed and analyzed by Tsurutani et al. (1997). Format is the same as in Fig. 1. Resolution is 1 sample/s. The MVAB eigenvectors, expressed in terms of their RTN components, and the corresponding variances are: $\hat{\boldsymbol{x}}_{1}=(-0.718 ; 0.058 ; 0.693)$ with $\lambda_{1}=0.0378 \mathrm{nT}$; $\hat{\boldsymbol{x}}_{2}=(-0.676 ;-0.293 ;-0.676)$ with $\lambda_{2}=0.129 \mathrm{nT}^{2} ; \hat{\boldsymbol{x}}_{3}=(0.164 ;-0.954 ; 0.250)$ with $\lambda_{3}=0.578 \mathrm{nT}^{2}$. In the bottom right panel, note that the slow rotation precedes the fast rotation for this event.

lower field magnitude on the fast branch than on the slow branch (see Fig. 10 in the work by Tsurutani and Ho, 1999). In both Figs. 8 and 9, the normal magnetic field is small but negative (anti-sunward). The more reliable result, which comes from the time interval of the rapid rotation only (see Table 1), is $\langle\boldsymbol{B}\rangle \cdot \boldsymbol{n}=(-0.42 \pm 0.02) \mathrm{nT}$; the corresponding normal vector from MVAB is $\boldsymbol{n}=(-0.853 ;-0.092 ;+0.513)$ (in the RTN system, where $R$ is radial outward from the sun, $N$ is due north in the plane containing $R$ and the sun's spin axis, and $T$ completes the right-handed orthogonal triad) and the eigenvalue ratio is $\lambda_{2} / \lambda_{1}=29$. Using this normal vector, but field vectors from the entire (slow + fast) event, we find $\langle\boldsymbol{B}\rangle \cdot \boldsymbol{n}=-0.68 \mathrm{nT}$ instead.
The low (242s) time resolution of the plasma data means that only four data points are available during the entire event. On the basis of these points one can calculate the average normal plasma flow in the HT frame to be positive, i.e., pointing towards the sun, $\left(\langle\boldsymbol{v}\rangle-\boldsymbol{V}_{\mathrm{HT}}\right) \cdot \boldsymbol{n}=$ $+12.8 \mathrm{~km} / \mathrm{s}$, which value is comparable to the Alfvén speed, $\left|V_{\mathrm{Ax}}\right|=11.8 \mathrm{~km} / \mathrm{s}$, based on the normal field $(-0.42 \mathrm{nT})$ and a proton density of $0.6 / \mathrm{cm}^{3}$. Also, the Walén slope is negative $(=-0.61$, with $c c=-0.982)$ as expected, although significantly less in magnitude than unity. The low slope may be the result mainly of the low time resolution but other effects, such as the presence of alpha particles, pressure anisotropy, and field fluctuations, are likely to play a role as well. The sunward flow in the proper (HT) frame of the 


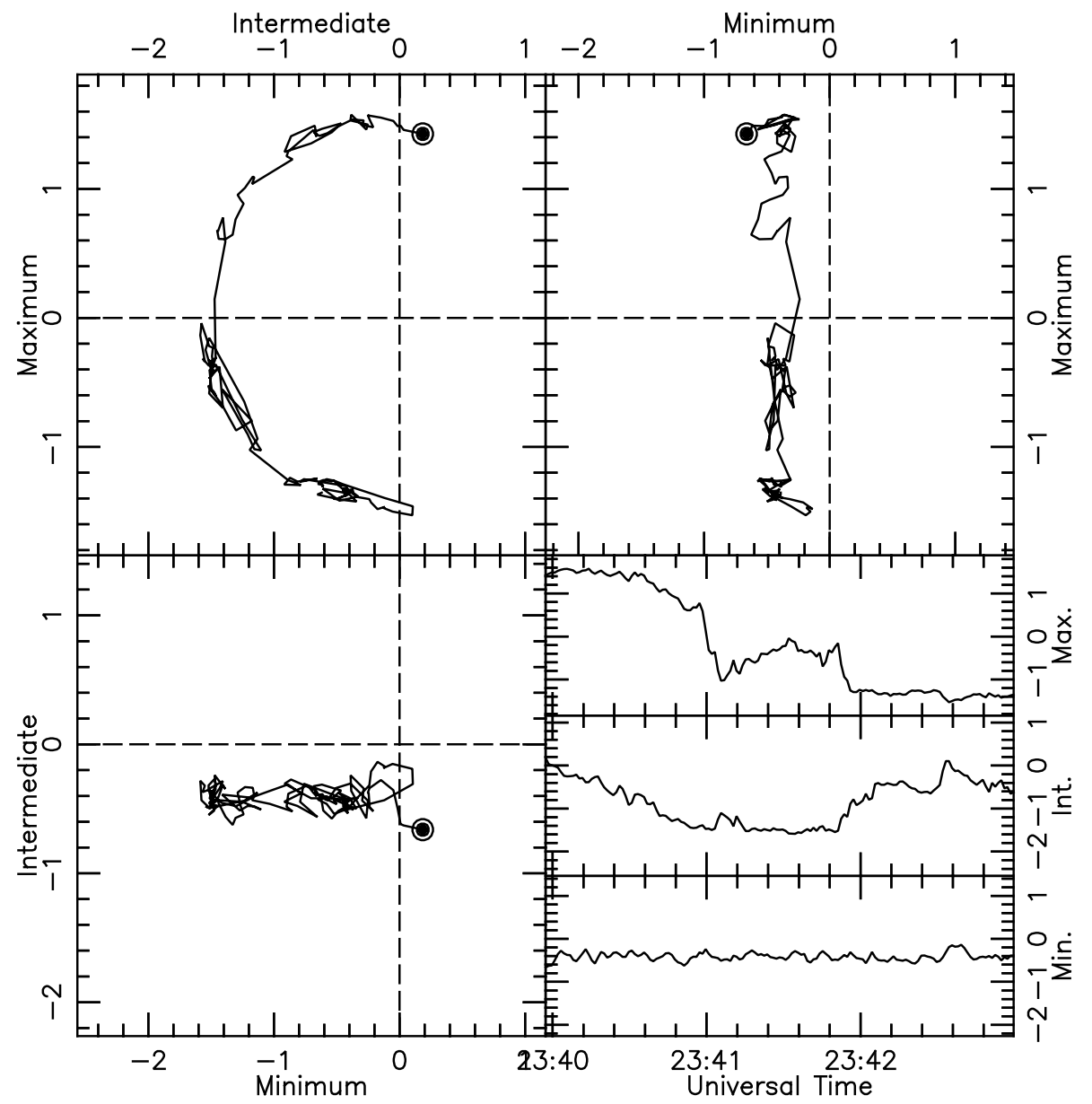

Time Interval (UT): 1995-07-29 23:40:47.710 - 23:43:19.720

Fig. 9. Ulysses event in Fig. 8: Hodogram for the rapid field rotation only. Resolution is 1 sample/s. The eigenvalue ratio is $\lambda_{2} / \lambda_{1}=29$; further details from the MVAB calculation are given in Table 1.

discontinuity indicates that, relative to the plasma, the structure was propagating outward from the sun, in agreement with the conclusion reached by Tsurutani and Ho (1999) from different considerations. The negative Walén slope is consistent with the fact that the normal field and the normal flow have opposite signs. This result also implies that the quantity $A_{\mathrm{x} 1}$ in Eq. (1) and Eq. (2) is negative and the negative sign in turn leads to a reversal of the predicted time order so that the outer (slow) rotation should now precede the inner (fast) rotation in the hodogram; this is indeed the observed behavior.

Although the statistical uncertainties are substantial, the internal consistency of the results supports our view that the signs of the nominal normal field and flow components are correct. An additional indication of the robustness of our result is that the signs of these components remain unchanged even if one uses the less accurate normal vector, obtained from MVAB on data for the entire event (Fig. 8), rather than from the rapid rotation only. This vector is $\boldsymbol{n}=(-0.719 ;+0.058 ;+0.692)$ with the much smaller eigenvalue ratio $\lambda_{2} / \lambda_{1}=3.4$; the corresponding normal field and flow are $\langle\boldsymbol{B}\rangle \cdot \boldsymbol{n}=-0.35 \mathrm{nT}$ and $\left(\langle\boldsymbol{v}\rangle-\boldsymbol{V}_{\mathrm{HT}}\right) \cdot \boldsymbol{n}=+8.8 \mathrm{~km} / \mathrm{s}$.

The durations of the rapid and the slow rotation are about $30 \mathrm{~s}$ and $928 \mathrm{~s}$, respectively. Since the structure moves antisunward at velocity $\boldsymbol{V}_{\mathrm{HT}} \cdot \boldsymbol{n}=680 \mathrm{~km} / \mathrm{s}$, the corresponding widths of the structure are about $20400 \mathrm{~km}$ and $631410 \mathrm{~km}$, respectively. These widths, seen at high latitude and a radial distance of $2 \mathrm{AU}$ from the sun, are an order of magnitude larger than those for the Cluster event, at low latitude and $1 \mathrm{AU}$. But because of the low ion density $\left(0.6\right.$ protons $\left./ \mathrm{cm}^{3}\right)$ at Ulysses, the ion inertial length is also larger $\left(\lambda_{i}=294 \mathrm{~km}\right)$ so that the rapid rotation occurs over a distance of about $70 \lambda_{i}$, which is roughly twice the result for Cluster $\left(26-32 \lambda_{i}\right)$. The difference could be accounted for by different values of 
$\left(A_{\mathrm{x}}^{2}-1\right)$ in the two events or by the two-step nature of the rapid field rotation (see Fig. 9). The result of the comparison of the two results can therefore be considered consistent with our claim that $\lambda_{i}$ is a key ingredient in the spatial scale of the rapid rotation.

\section{Summary and discussion}

In this paper, we have shown that the isentropic/polytropic Hall-MHD equations have exact 1-D solutions describing infinite, plane, nonlinear wave trains in which the magnetic field transverse to the propagation direction rotates back and forth, within an angle range of up to $\pm 180^{\circ}$, with a nearly, but not precisely, constant field magnitude. The magnetic field component in the propagation direction is constant and is usually small. We have then compared the theoretically predicted behavior during one wave period with behavior of one double-arc polarized structure observed by the four Cluster spacecraft and one observed by the Ulysses spacecraft. The observations show fast field rotation in one sense of direction with slightly weaker magnetic field, followed by (for the Cluster event), or preceded by (for the Ulysses event), a much slower rotation in the opposite sense with a slightly larger field magnitude. Although our theoretical model does not account for the difference in rotation rates, it appears to well describe many of the other observed features. This leads us to conclude that the slow rotation observed adjacent to the fast rotation is not a separate phenomenon but is instead an integral part of an overall propagating wave structure. The fast or slow rotation part can be separately called either a rotational discontinuity (RD) or an arc-polarized directional discontinuity (although the term "discontinuity" is less apt for the slow part). For clarity, we have referred to the overall (fast + slow) structure as being double-arc polarized. We have shown that our model can describe the fast rotation part, including its width, as well as the average field behavior in the slow rotation, except for the slow rotation rate. The model does not describe the field fluctuations seen during the slow rotation; these fluctuations are not consistent with the 1-D, time-independent nature of the model. Finally, we emphasize that solutions of the type we have found do not exist in ordinary MHD; the Hall term in the generalized Ohm's law, and with it the ion inertial length $\lambda_{i}$, plays a critical role. We have also shown that the characteristic length scale is $\lambda_{i} /\left(A_{\mathrm{x}}^{2}-1\right)$, where $A_{\mathrm{x}}=v_{\mathrm{x}}\left(\mu_{0} n m_{i}\right)^{1 / 2} / B_{\mathrm{x}}$ is the Alfvén number based on the plasma flow component (in the co-moving frame) and the field component along the x-axis, i.e., in the direction normal to the wave fronts. Provided $A_{\mathrm{x}}^{2}$ has values sufficiently close to one, the characteristic length scale, i.e., the wavelength, can therefore be many $\lambda_{i}$.

The detailed features of the theoretical model and their relationship to observed features, and to basic physics, can be summarized as follows.
1. The tangential hodogram for the wave has two branches: an inner branch in which the average field magnitude is slightly depressed and the Alfvén number $A_{\mathrm{x}}$ has magnitude slightly less than one; an outer branch, in which the field magnitude is slightly enhanced and the magnitude of $A_{\mathrm{x}}$ is slightly larger than one. The separation of the two branches depends on the chosen parameter values, including the net rotation angle of the tangential magnetic field. It is usually small. These features of the model are present in hodograms from real physical events observed in the solar wind (Figs. 1 and 8). One usually sees only one full period of the wave train; this period starts and ends with the tangential field in, or near, an extreme position, at one of the two turning points of the hodogram. In our model, the turning points are not fixed points and therefore do not represent the precise asymptotic upstream or downstream state of a discontinuity. But it is around the turning points that the field rotation rate is small, making possible, via small disturbances, the creation of a local fixed point (a center) and therefore a transition to a small amplitude gyration or periodic tipping back and forth around a field representative of neighboring solar-wind regions. The same argument indicates that observations of only one part of a full period, from turning point to turning point along either the inner or outer branch, should occur as well. These are the single-arc polarized structures referred to as rotational discontinuities (RDs). Comparison of their predicted properties with observations will be presented in a separate paper. Temporal variations can probably also lead to the creation of transient fixed points elsewhere along the hodogram curve. Such behavior appears to be present on the outer hodogram branch (e.g., Fig. 1). It can in principle produce extremely complicated hodogram structures, as frequently seen in the observations.

2. As seen by an observer traveling with a plasma element across the structure, the predicted field rotation on the outer branch is always electron polarized, i.e., the rotation sense is the same as that of an electron gyrating in the normal component of the magnetic field. Similarly, the predicted rotation on the inner branch is always ion polarized. As discussed below, the observations indeed show this behavior. Since an ion-polarized whistler (regardless of amplitude) has a phase velocity that is increasingly less than the Alfvén speed as the wavelength shortens, such a wave can phase stand in the flow only if the local conditions have $A_{\mathrm{x}}^{2}<1$; this is the situation on the inner branch of the hodogram. Similarly, the electron-polarized whistler at long wavelengths has phase velocity larger than the Alfvén speed; to phase stand, such a wave must be in a flow where $A_{\mathrm{x}}^{2}>1$, as is indeed the case on the outer hodogram branch. 
Because mass conservation requires $\rho v_{\mathrm{x}}=$ const., a larger (smaller) value of $A_{\mathrm{x}}^{2}=\left(\rho v_{\mathrm{x}} / B_{\mathrm{x}}\right)^{2} \mu_{0} / \rho$ corresponds to a lower (higher) density and, from the isentropic/polytropic law $\left(p / \rho^{\gamma}=\right.$ const.), a lower (higher) plasma pressure and a higher (lower) ram pressure $\rho v_{\mathrm{x}}^{2}$. As long as $\gamma>1$, a lower (higher) plasma pressure $p$ wins over the higher (lower) ram pressure in the normal stress balance, $\left(\rho v_{\mathrm{x}}^{2}+p+B_{\mathrm{t}}^{2} / 2 \mu_{0}\right)=$ const. To maintain the overall balance, the tangential magnetic field must therefore be stronger on the electron-polarized branch and weaker on the ion-polarized branch, which is indeed the case. Thus the separation of the two branches is a direct consequence of the dispersive properties of whistler waves, which properties result from the inclusion of the Hall term in Ohm's law. Furthermore, the separation of the branches increases as $\gamma$ increases. In the MHD limit, $A_{\mathrm{x}}^{2} \rightarrow 1$ on both branches, which then coincide, but the price paid is that the wavelength $\rightarrow \infty$, which means that the field rotation rate becomes infinitely slow. In this limit, the fixed point (point 2 in Fig. 2a), around which our hodogram traces are nested, degenerates into a circle on which $A_{\mathrm{x}}^{2}=1$; contrary to the situation depicted in Fig. 2a, this circle is now centered exactly at the origin $(0,0)$ of the tangential hodogram plane.

3. When, as in our Cluster event, the normal field component and the normal flow component have the same sign (either ++ or -- ), and a period of the wave structure is taken to start at the largest negative value of the field angle $\psi=\tan ^{-1}\left(B_{\mathrm{y}} / B_{\mathrm{z}}\right)$, i.e., at the left turning point in Fig. 2a, then an observing spacecraft will see the tip of the tangential field vector start moving to the right along the inner branch of the hodogram. This ion-polarized rotation takes the tangential field to its most positive $\psi$ value. After the right turning point has been reached, an electron-polarized rotation follows along the outer branch, back to the original $\psi$ value. In the theoretical model, this return rotation has about the same thickness as the rotation on the inner branch but, as observed by Cluster, it is $34-43$ times wider. In this event, what is seen by an observing spacecraft as the structure is carried past it by the solar wind, is therefore a rapid rotation followed by a slow return rotation (Figs. 1 and 7). The observed rapid rotation occurs over a distance (thickness) along the propagation direction of some 2632 ion inertial lengths.

When, as in the Ulysses event, the normal field and flow components have opposite signs instead (either +- or $-+)$, the spacecraft will first see a slow rotation followed by a rapid return rotation (see the time plot in Fig. 8). In the Ulysses event, the thickness of the rapid rotation was about 70 ion inertial lengths and the width of the slow rotation was some 31 times larger.
Note that the sense of field rotation recorded in a measured hodogram agrees with the sense that would be seen by an observer traveling with the plasma across the structure only if the propagation direction is away from the sun; if it is toward the sun, the sense is reversed. In the Ulysses, $\mathrm{C} 1$, and $\mathrm{C} 3$ crossings, the former was the case; for $\mathrm{C} 2$ and $\mathrm{C} 4$, the latter situation occurred.

4. Since the sign of the normal flow component in the HT frame determines the propagation direction of the structure relative to the plasma, we conclude that the propagation sense can be determined from magnetic data alone. For a fast-slow event, where, additionally, the sign of the normal magnetic field component can be established with confidence (from MVAB or otherwise), a positive (sunward) value of this field component implies that the structure propagates anti-sunward, while a negative (anti-sunward) value means it propagates sunward. The former case was found for the $\mathrm{C} 1$ and $\mathrm{C} 3$ observations of our Cluster event and the latter was the case for the slightly earlier $\mathrm{C} 2$ and $\mathrm{C} 4$ observations. By use of CIS/HIA data, we found that the sign of the predicted normal flow was indeed positive for $\mathrm{C} 1$ and $\mathrm{C} 3$. This result may be fortuitous but it was consistent with the positive Walén slope during the entire event. For C2, the entire CIS instrument is non-functional. Therefore, the predicted sunward propagation direction could not be checked. For C4, the CIS/CODIF instrument is functional and confirms the predicted sunward propagation and the positive Walén slope. Because it is hard to imagine a physical process that would rapidly reverse the sign of the Walén slope, we conclude it must have been positive for $\mathrm{C} 2$ as well. This conclusion in turn confirms that the propagation direction was sunward during the $\mathrm{C} 4$ and $\mathrm{C} 2$ crossings. For a slow-fast case, such as the Ulysses event, the normal field and flow should have opposite signs and, since the normal field was found to be negative (anti-sunward), the normal flow should be positive (sunward), corresponding to anti-sunward propagation. This prediction was directly, albeit perhaps fortuitously, confirmed by use of data from the plasma instrument, which data indicated sunward flow along the normal direction as well as the expected negative Walén slope. Tsurutani and Ho (1999) also found anti-sunward propagation. Note that the rapid rotation is not always at the leading (inflow) side of the wave, with the slow rotation on the trailing (outflow) side. It is evident from the Cluster 2 and 4 crossings and from the Ulysses event that such is not the case: the slow rotation is sometimes on the leading side of the structure and the rapid rotation on the trailing side. Therefore an analogy with wind-driven water waves cannot be drawn.

5. From the discussion in items 3 and 4 follows that there is a one-to-one correspondence between the sign of the Walén slope and the sense of motion along the 
hodogram trace. When the slope is positive, so that $v_{\mathrm{x}}$ and $B_{\mathrm{x}}$ have the same signs (either ++ or -- ), the order seen in the hodogram is fast (inner-branch) rotation followed by slow (outer-branch) rotation. When the Walén slope is negative, so that $v_{\mathrm{x}}$ and $B_{\mathrm{x}}$ have opposite signs (either +- or -+ ), the order seen is slow rotation followed by fast rotation. It follows that one can replace information about the Walén slope, for which plasma measurements are required, by simple observation of the ordering of the fast (inner-branch) and slow (outer-branch) rotation parts of an event, which requires only the field measurements. To determine the actual propagation direction, i.e., to find the sign of $v_{\mathrm{x}}$, one can either use direct plasma measurements of that velocity component, or one can determine the sign of $B_{\mathrm{x}}$. When these components are small, both determinations are difficult, but reliably establishing the sign of $B_{\mathrm{x}}$ tends to be the easier task.

6. In order to match the theoretical model to the branch separation in the $\mathrm{C} 1$ hodogram and at the same time to the spatial width of the inner branch, it was necessary to use a $\gamma$ value smaller than that of isentropic behavior, converting the model to a polytropic one. This corruption of the ideal physical model is an indication that processes and effects not included in the model in reality play a non-negligible role. A further indication that something is missing is that the Walén slope magnitudes are significantly less than unity.

7. For the Cluster event, the "strahl" electron flow was found to be anti-parallel to the magnetic field. For $\mathrm{C} 1$ and $\mathrm{C} 3$, where the normal field pointed toward the sun, this fact could perhaps be taken to suggest that the sunward facing side of the structure was magnetically connected to the sun in a direct way (the field lines had one "end" on the solar surface), whereas the earthward facing side was so connected only via the small sunward pointing (positive) normal magnetic field component across the structure. However, if the same argument were applied to the $\mathrm{C} 2$ and $\mathrm{C} 4$ observations instead, the conclusion would be the reverse: The earthward side would be directly connected and the sunward side only via the small, now earthward pointing (negative) normal field. It appears that, on and off, both sides must have had direct connection to perhaps different regions on the solar surface. The implication is that "strahl" information cannot be used to unambiguously decide that one side of a discontinuity has direct connection and the other one only indirect connection to the sun.

8. We now comment on the failure of the model to account for the slowness of the field rotation on the outer, electron-polarized branch of the hodogram. We have not studied the stability of our solution and doing so is not a simple matter. One possibility is that, at least for the parameter values applicable to our events, the electron-polarized part of our theoretically derived structure (in which part the group velocity exceeds the phase velocity) is unstable and can spread out over time. In the solar wind, the corresponding situation would be that, as the overall structure moves outward from the sun, the ion-polarized part (in which the group velocity is less than the phase velocity) is able to reach its equilibrium thickness but the electron-polarized part is not. At least the electron-polarized part of the observed structures may therefore be evolving with time. Furthermore, one can perhaps understand how the ionpolarized part can steepen when it is located on the upstream side of the wave structure (as for $\mathrm{C} 1$ and $\mathrm{C} 3$ ): ion polarized wave packets generated within the structure cannot escape upstream. But it is not clear how it can happen when this part is on the downstream side (as for $\mathrm{C} 2, \mathrm{C} 4$, and Ulysses).

Many studies of the evolution of Alfvén waves as they are convected away from the sun can be found in the literature. A detailed discussion of these is beyond the scope of our paper. We simply note that $1 \frac{1}{2}$-D hybrid simulations (Vasquez and Hollweg, 1996) of the evolution of linearly polarized Alfvén waves near the sun into arc-polarized wave trains do not show the difference between fast and slow field rotation rates found in the observed events; the behavior they find is instead somewhat similar to that shown in our Fig. 4. Noticeable differences are that, in the simulation results, the field rotation rate is slower around the turning points and that there is no readily noticeable separation between the two branches of the hodogram. The parameter values used in the simulations were similar to those of the Cluster event, except that the propagation angle relative to the magnetic field was smaller $\left(\theta_{B}=60^{\circ}\right.$ versus $\theta_{B} \simeq 86.2^{\circ}$ for Cluster). It must be remembered that the simulations, as well as our equilibrium solution, by no means incorporate all features of the real situation, such as global-scale gradients and associated deformations of flow and field, the presence of alpha particles, and the presence of deviations from 1-D behavior. We also cannot exclude the possibility that events may be found in which the field rotation for the electronpolarized branch is as well ordered and rapid as it is for the ion-polarized branch.

There are 1-D processes, such as modulation and decay instabilities that could play a role. But in the Vasquez and Hollweg (1986) simulations, these instabilities, if at all present, do not seem to produce the observed different rotation rates for the ion- and electron-polarized parts of a double-arc event. Perhaps the explanation lies in the periodic nature of the simulation. If only a single period were included, it is possible 
that the observed behavior might develop, as in the article by Spangler et al. (1985). Since this latter work is based on weakly nonlinear analysis, it is not clear how relevant it is to our case, where the wave amplitude $\left(B_{\mathrm{t}} / B_{\mathrm{x}}\right)$ is very large.

Another possibility is that the flaw lies in the 1-D nature of both simulation and equilibrium model, although subsequent $2 \frac{1}{2}$-D simulations (Vasquez and Hollweg, 1998a, b) also do not appear to slow down the electronpolarized field rotation. Perhaps the large amplitude fluctuations seen on this branch are a signature and essential ingredient of the process that leads to the slow average rotation rate in the electron-polarized portion of a wave period. These fluctuations are essentially Alfvénic and propagate along the local, slowly rotating, average tangential field and causing the field vector to tip back and forth along the slow hodogram branch. In the $1 \frac{1}{2}$-D simulations and in our model, such fluctuations cannot occur because they also involve fluctuations of the normal field component. In the model, their influence could perhaps be described in an average sense by inclusion of some extra terms in the conservation laws or by using slightly different parameter values on the outer branch. In this context, we note that the field rotation rate becomes smaller the closer the branch comes to the circle defining Alfvénic normal flow.

It is also possible that the fluctuations do not play a significant role but are simply part of a large sea of Alfvén waves in which the structure is immersed. There is time for these waves to show up during the slow rotation, while the duration of the fast rotation may usually be too short for their presence to be evident. However, in the Ulysses hodogram (Fig. 9), there are in fact indications that these fluctuations can occur also during the fast part of the structure, causing it to have longer duration. Unfortunately, the overall conclusion is that the true reason for the observed slow field rotation on the outer hodogram branch remains unknown.

9. For the Cluster event, a second mystifying feature is the reversal of the inferred propagation direction from sunward for the earliest (C4) and the second earliest (C2) crossing to anti-sunward for the next-to-last (C3) and the last (C1) crossing (see Fig. 7). The near simultaneity of the $\mathrm{C} 2$ and $\mathrm{C} 3$ crossings indicates that the reversal must be the result of spatial effects on the scale of the spacecraft separation. Further evidence of pronounced spatial structure is provided by the fact that the four MVAB normal vectors, based on high resolution data during the rapid parts of the structure, all have high quality, as judged by the eigenvalue ratio, but yet point in widely different directions, as shown in Fig. 6 . The conclusion is again that there must be substantial spatial structure on the scale of the spacecraft separation and presumably also associated temporal evolution.
The reasons for these effects are not obvious but could involve Kelvin-Helmholtz and/or tearing mode instabilities operating mainly in the relatively thin ion-polarized part of the structure, or it could be a consequence of the fluctuations seen during the slow rotation. As shown in Fig. 6, the normal vectors from the full event, which are determined mainly by the data from the slow rotation, have less spread. The results as they stand seem to torpedo the concept that double-arc polarized magnetic structures are always nice and one-dimensional over substantial spatial scales. A consequence is that a normal vector determination from relative timing of the four crossings by use of the standard constant velocity assumption (CVA; see the article by Haaland et al., 2004), while providing perhaps a reasonable average orientation (see Fig. 6), cannot always be used to calculate meaningful values of the small normal magneticfield and flow components at each individual crossing.

Acknowledgements. The research reported here results from a team effort, sponsored by the International Space Science Institute (ISSI), Bern, Switzerland, and devoted to the study of solar-wind directional discontinuities. We thank ISSI for its support and the other team members, T. Horbury, W.-L. Teh, J. Vogt, and R. Wicks, for helpful comments. We also thank A. Balogh and E. Lucek for use of the Cluster field data; H. Rème and I. Dandouras, for the Cluster plasma data; A. Fazakerley for the Cluster electron data; A. Balogh for the Ulysses field data; and D. J. McComas, for the Ulysses plasma data as well as the ACE data concerning the alpha/proton ratio.

Topical Editor I. A. Daglis thanks M. Goldstein and E. Podladchikova for their help in evaluating this paper.

\section{References}

Haaland, S. E., Sonnerup, B. U. Ö., Dunlop, M. W., Balogh, A., Georgescu, E., Hasegawa, H., Klecker, B., Paschmann, G., PuhlQuinn, P., Rème, H., Vaith, H., and Vaivads, A.: Four-spacecraft determination of magnetopause orientation, motion and thickness: comparison with results from single-spacecraft methods, Ann. Geophys., 22, 1347-1365, 2004, http://www.ann-geophys.net/22/1347/2004/.

Hau, L.-N. and Sonnerup, B. U. Ö.: On the structure of resistive intermediate shocks, J. Geophys. Res., 94, 6539-6551, 1989.

Hau, L.-N. and Sonnerup, B. U. Ö.: The structure of resistivedispersive intermediate shocks, J. Geophys. Res., 95, 1879118808, 1990.

Horbury, T. and Tsurutani, B. T.: Ulysses measurements of waves, turbulence and discontinuities, Chapter 4, pp 167-227, in: The Heliosphere Near Solar Minimum, edited by: Balogh, A., Marsden, R. G., and Smith, E. J., Springer Verlag, Berlin, ISBN 185233-204-2, 2001.

Khrabrov, A. V. and Sonnerup, B. U. Ö.: DeHoffmann-Teller Analysis, Ch. 9, pp 221-248, in: Analysis Methods for MultiSpacecraft Data, edited by: Paschmann, G. and Daly, P. W., ISSI SR-001, ESA Publ. Div. Noordwijk, 1998. 
Lichtenstein, B. R. and Sonett, C. P.: Dynamic magnetic structure of large amplitude Alfvénic variations in the solar wind, Geophys. Res. Lett., 7, 189-192, 1980.

Riley, P., Sonett, C. P., Tsurutani, B. T., Balogh, A., Forsyth, R. J., and Hoogeveen, G. W.: Properties of arc-polarized Alfvén waves in the ecliptic plane: Ulysses observations, J. Geophys. Res., 101, 19987-19993, 1996.

Sonnerup, B. U. Ö. and Scheible, M.: Minimum and maximum variance analysis, Ch. 8, pp 185-220, in: Analysis Methods for Multi-Spacecraft Data, edited by: Paschmann, G. and Daly, P. W., ISSI SR-001, ESA Publ. Div. Noordwijk, 1998.

Spangler, S. R., Sheerin, J. P., and Payne, G. L.: A numerical study of nonlinear Alfvén waves and solitons, Phys. Fluids, 28, 104109, 1985.

Tsurutani, B. T., Ho, C. M., Smith, E. J., Neugebauer, M., Goldstein, B. E., Mok, J. S., Arballo, J. K., Balogh, A., Southwood, D. J., and Feldman, W. C.: The relationship between interplanetary discontinuities and Alfvén waves: Ulysses observations, Geophys Res. Lett., 21, 2267-2270, 1994.

Tsurutani, B. T., Ho, C. M., Arballo, J. K., Smith, E. J., Goldstein, B. E., Neugebauer, M., Balogh, A., and Feldman, W. C.: Interplanetary discontinuities and Alfvén waves at high heliographic latitudes: Ulysses, J. Geophys. Res., 101, 11027-11038, 1996.
Tsurutani, B. T., Ho, C. M., Arballo, J. K., Lakhina, G. S., Glassmeier, K.-H., and Neubauer, F. M.: Nonlinear electromagnetic waves and spherical arc-polarized waves in space plasmas, Plasma Phys. Contr. Fusion, 39(A5), A237-A250, 1997.

Tsurutani, B. T. and Ho, C. M.: A review of discontinuities and Alfvén waves in interplanetary space: Ulysses results, Rev. Geophys., 37(4), 517-541, 1999.

Vasquez, B. J. and Hollweg, J. V.: Formation of arc-shaped Alfvén waves and rotational discontinuities from oblique linearly polarized wave trains, J. Geophys. Res., 101, 13527-13540, 1996.

Vasquez, B. J. and Hollweg, J. V.: Formation of spherically polarized Alfvén waves and embedded rotational discontinuities from a small number of entirely oblique waves, J. Geophys. Res., 103, 335-347, 1998a.

Vasquez, B. J. and Hollweg, J. V.: Formation of embedded rotational discontinuities with nearly field-aligned normals, J. Geophys. Res., 103, 349-365, 1998b. 